Submitted to J. Geophys. Res.: June 1, 2004.

Revised: August 18, 2004.

\title{
Stratification of Anisotropy in the Pacific Upper Mantle
}

\author{
Daniel B. Smith, Michael H. Ritzwoller†, Nikolai M. Shapiro
}

Center for Imaging the Earth's Interior

Department of Physics

University of Colorado

Campus Box 390, Boulder, CO 80309-0390, USA

$\dagger$ To whom correspondence should be directed: ritzwoller@ciei.colorado.edu

\begin{abstract}
Based on the use of broad-band (25 sec - $150 \mathrm{sec}$ ) Rayleigh wave group speeds to estimate the $2 \psi$ component of azimuthal anisotropy, we present evidence for a stratification of anisotropy in the uppermost mantle at large-scales across the Pacific basin. We confirm previous surface wave studies that established that the fast-axis directions of azimuthal anisotropy for intermediate and long period Rayleigh waves approximately align with present-day plate motions. At shorter periods (25 sec - $50 \mathrm{sec}$ ), however, fast-axes align nearer to the paleo- or fossil spreading direction which differs from present-day plate motion in the old Pacific. These observations, as well as observations of the age dependence of the amplitude of azimuthal anisotropy, imply that azimuthal anisotropy in the Pacific upper lithosphere $(<100 \mathrm{~km}$ depth) is fixed or "fossilized", on average, reflecting the strain conditions extant during the early evolution of the lithosphere rather than the current ambient flow direction. In the deeper lithosphere and asthenosphere, anisotropic fast axis directions align nearer to present-day plate motions, apparently having re-oriented to conform to the current conditions of mantle flow. The mechanism of anisotropy stratification remains unclear, but observations are consistent with the anisotropy of the shallow lithosphere being fixed because the shear flows that can produce dislocation creep and a change in anisotropy will occur at increasing depths as the plate ages.
\end{abstract}




\section{Introduction}

A large body of evidence has established that seismic wave propagation in the Earth's uppermost mantle is anisotropic. At large scales, much of this evidence has derived from surface wave dispersion, although anisotropy in $S_{n}$ and $P_{n}$ travel times has played a centrally important role in establishing the anisotropic character of the oceanic upper mantle (e.g., Hess, 1964; Raitt et al., 1969; Shearer and Orcutt, 1986) and strenuous efforts have been devoted to the study of shear wave splitting to image smaller-scale variations in anisotropic structure (e.g., Savage, 1999 for a review).

There are two general types of surface wave anisotropy that have been observed. The first is that vertically polarized surface waves propagate more slowly than horizontally polarized waves in the uppermost mantle (e.g., Aki and Kaminuma, 1963; McEvilly, 1964, and many others) and regionally in the crust (e.g., Shapiro et al., 2004). This "Rayleigh-Love discrepancy" indirectly implies widespread radial anisotropy (transverse isotropy with a vertical symmetry axis) whose strength varies over the Earth's surface (e.g., Ekström and Dziewonski, 1998). Radial anisotropy has been a common feature of both 1-D (e.g., Dziewonski and Anderson, 1981) and 3-D (e.g., Nataf et al., 1984) mantle models for the past two decades. The second type of surface wave anisotropy is the variation of surface phase speeds with azimuth (e.g., Forsyth, 1975; Nishimura and Forsyth, 1989; Montagner and Tanimoto, 1991; Ekström, 2000; Trampert and Woodhouse, 2003) which provides direct evidence for azimuthal anisotropy where the fast axis of propagation lies in the horizontal plane. Inverting for azimuthal anisotropy is difficult, however, as the variables that describe it must be introduced to an isotropic inverse problem which is already underdetermined. The amplitude of the azimuthal terms depends strongly on the damping and regularization applied to the inversion (e.g., Barmin et al., 2001), which makes the strength of azimuthal anisotropy, in particular, difficult to estimate. Inferences, therefore, must necessarily concentrate on patterns of azimuthal anisotropy and perhaps relative lateral amplitude variations.

Radial and azimuthal anisotropy of surface waves have a common cause, the underlying anisotropy of rocks in the Earth's mantle. Anisotropy in upper mantle rocks can be explained by the accumulation of strain due to mantle convection (e.g., McKenzie, 1979) which manifests itself as lattice preferred orientation (LPO) of mantle minerals, in particular olivine (e.g., 
Babuska and Cara, 1991, for a review). This clustering of crystallographic axes around a particular orientation is typically referred to as mineralogical fabric. Only a modest alignment of olivine is needed to produce commonly observed mantle anisotropy strengths of $2-6 \%$ (Ribe and Yu, 1992). The interest in seismic anisotropy, therefore, is that, at least in principal, observations of anisotropy can be used to constrain mantle flow (e.g., McKenzie, 1979; Regan and Anderson, 1984; Tanimoto and Anderson, 1984; Ribe, 1989; Chastel et al., 1993; Russo and Silver, 1994; Tommasi, 1998; Buttles and Olson, 1998; Hall et al., 2000; Blackman and Kendall, 2002; Blackman et al., 2002; Becker et al., 2003; Gaboret et al., 2003). To date, the most straightforward results have been obtained in relatively young oceanic areas where the direction of the fast axis of azimuthal anisotropy is approximately aligned with the spreading direction (e.g., Hess, 1964; Raitt et al., 1969; Morris et al., 1969; Shearer and Orcutt, 1986; Wolfe and Solomon, 1998). This alignment has been taken as evidence for the near-horizontal shearing of the uppermost oceanic mantle in the direction of plate motion, approximately perpendicular to the mid-oceanic ridge. This idea is supported by theoretical studies (e.g., Ribe, 1992; Kaminski and Ribe, 2001; Blackman et al., 2002) and laboratory measurements (e.g., Zhang and Karato, 1995) suggesting that the fast axis of olivine tends to align nearly parallel to the direction of shear.

These results have led to a simple model that postulates that the observed fast axis of seismic anisotropy beneath oceans is, on average, close to the the direction of shear or to the largest axis of the finite-strain ellipsoid associated with on-going mantle flow. Under this interpretation, azimuthal anisotropy can be used as proxy for the pattern of mantle flow and observations of shear-wave splitting (e.g. Russo and Silver, 1994; Peyton et al., 2001; Silver and Holt, 2002) and surface-wave azimuthal anisotropy (e.g., Gaboret et al., 2003; Becker et al., 2003) have been used to infer regional and global flow patterns.

There are several reasons to be cautious with this postulated interpretation of seismic anisotropy as a proxy for mantle flow. First, evidence for a depth-dependent distribution of anisotropy has been found both beneath continents (e.g., Savage and Silver, 1994; Levin et al., 1999; Vinnik et al., 2002) and oceans (e.g., Wolfe and Solomon, 1998). In addition, in most areas the observed seismic anisotropy is more complicated than a system with a fast horizontal axis of symmetry and may require additional radial anisotropy which has a vertical slow axis 
of symmetry (e.g., Ekström and Dziewonski, 1998; Shapiro and Ritzwoller, 2002). Second, the formation and evolution of mantle anisotropy in response to deformation is a complex process that depends on the thermal history and composition of the upper mantle. It has been argued, for example, that some observations of seismic anisotropy are attributable to fossil mineralogical fabric (e.g., Wolfe and Solomon, 1998) or to the presence of water in the upper mantle that can result in a fast anisotropy axis nearly perpendicular to the flow direction (e.g., Jung and Karato, 2001).

This paper aims to test the hypothesis that at large scales azimuthal anisotropy can be used as an indicator for mantle flow where, in particular, we are interested if anisotropy stands in a simple geometrical relation to sub-lithospheric shearing in the direction of present or past plate motions. A corollary to this hypothesis is whether water in upper mantle minerals is strongly affecting the relation between mantle flow and seismic anisotropy at large-scales. We estimate the distribution of azimuthal anisotropy across the Pacific by inverting surface-wave dispersion data. We focus on the Pacific for three reasons. First, because the oceanic crust is thin the anisotropy of oceanic crustal minerals does not obscure estimates of mantle anisotropy as strongly as does the anisotropy of the much thicker continental crust. Second, both the present-day plate kinematics and the past tectonic history of the Pacific are relatively well understood. Third, the natural distribution of seismicity around the Pacific provides relatively good path and azimuthal coverage and, as we will show below, robust large-scale patterns of azimuthal anisotropy can be inferred from the inversion of surface waves crossing the Pacific. Figure 1a shows a bold black contour that defines what we refer to as "the Pacific". It includes parts of the Nazca, Cocos, Juan de Fuca and Antarctic Plates in addition to most of the Pacific Plate where lithospheric age is well determined (Mueller et al., 1997).

There have already been numerous studies of surface-wave azimuthal anisotropy across the Pacific (e.g., Forsyth, 1975; Nishimura and Forsyth, 1988, 1989; Montagner and Tanimoto, 1991; Montagner, 1998; Ekström, 2000; Trampert and Woodhouse, 2003; Becker et al., 2003). These studies generally agree that in the young Pacific anisotropic fast axes tend to align with present-day plate motions, lying nearly perpendicular to the East Pacific Rise. In addition, Nishimura and Forsyth $(1988,1989)$ present evidence that in the old, western Pacific fast axis tend to align nearer to the paleo-spreading direction. The more recent studies with large 
numbers of crossing paths across the Pacific have been based on relatively long period (greater than $\sim 40 \mathrm{sec})$ surface wave phase speeds which provide rather poor vertical resolution. The earlier studies based on a coarser data set, in contrast, extended analyses to periods as low as $20 \mathrm{sec}$ (Nishimura and Forsyth, 1988, 1989) and included measurements of both group and phase speeds (e.g., Forsyth, 1975). In the present paper, we apply a large, newly compiled set of group speed observations that are measured down to periods of $\sim 20$ sec, which improves both lateral and depth resolution in the uppermost mantle relative to other studies.

As described below, we confirm recent surface wave studies that established that the fast-axis directions of azimuthal anisotropy for intermediate and long period $(>\sim 50 \mathrm{sec})$ Rayleigh waves approximately align with present-day plate motions. We show, however, that at shorter periods fast-axes align nearer to the paleo-spreading direction as hypothesized by Nishimura and Forsyth $(1988,1989)$. This suggests that anisotropy in the shallow upper mantle is "frozen in" or "fossilized" and represents the conditions of formation of the lithosphere rather than the current ambient flow direction. Below the shallow lithosphere, fast axis directions more nearly conform to present-day plate motions. A two-layered system of anisotropy also has been suggested to explain observations of shear-wave splitting near Hawaii (Wolfe and Solomon, 1998), although in that study the orientation of the deeper anisotropy is postulated to be related to return flow to the Pacific Superswell.

In section 2 , we describe the group velocity data set and the method of inversion used to produce maps of azimuthal anisotropy across the Pacific. The results of the inversion are presented in section 3 and their meaning is discussed in section 4 .

\section{Data and Method of Inversion}

\subsection{Data}

The data set consists of Rayleigh wave group speed measurements obtained from global and regional networks. Ritzwoller and Levshin (1998) describe the data selection and group velocity measurement procedures. Globally, measurements have been obtained for more than 200,000 individual minor arc paths. There are no group velocity measurements for major arc paths. After a preliminary elimination of outliers based on summary-ray analysis, the data are "cleaned" by rejecting measurements whose observed travel times disagree with travel 
times predicted by the 3-D model of Shapiro and Ritzwoller (2002) by more than three times the rms-misfit over the entire data set. About $98 \%$ of the original observations are retained, resulting in $28,800,28,100,19,700$, and 11,600 group velocity measurements at periods of 25 , 50, 100, and $150 \mathrm{sec}$, respectively, that cross some part of the Pacific basin. The resulting path density is shown in Figure 1. Path density degrades appreciably above about 100 sec period and is regionally worst in the central, eastern Pacific. Path density over large expanses of the very young and the very old Pacific, which we will contrast and on which we will concentrate interpretation, is adequate, however, except arguably at very long periods.

\subsection{Surface Wave Tomography}

The azimuthal dependence of local phase (Smith and Dahlen, 1973, 1975; Romanowicz and Snieder, 1988; Larsen et al., 1998) and group speed in the presence of a weak general anisotropy is given by

$$
\begin{aligned}
c(\mathbf{r}, \psi) & =c_{I}(\mathbf{r})+c_{A}(\mathbf{r}, \psi) \\
c_{I}(\mathbf{r}) & =A_{0}(\mathbf{r}) \\
c_{A}(\mathbf{r}, \psi) & =A_{1}(\mathbf{r}) \cos (2 \psi)+A_{2}(\mathbf{r}) \sin (2 \psi)+A_{3}(\mathbf{r}) \cos (4 \psi)+A_{4}(\mathbf{r}) \sin (4 \psi),
\end{aligned}
$$

where $\mathbf{r}$ is the position vector, $c_{I}$ and $c_{A}$ are the local isotropic and azimuthally anisotropic parts of the wave speed, respectively, $A_{0}$ is the isotropic coefficient, $A_{1}, \ldots, A_{4}$ are the anisotropic coefficients, and $\psi$ is the local azimuth. The coefficients $A_{0}, \ldots, A_{4}$ depend on

period and wave type (Rayleigh/Love). The tomography procedure to estimate $A_{0}, \ldots, A_{4}$ is described by Barmin et al., (2001). The method minimizes a penalty functional that can be represented schematically as follows:

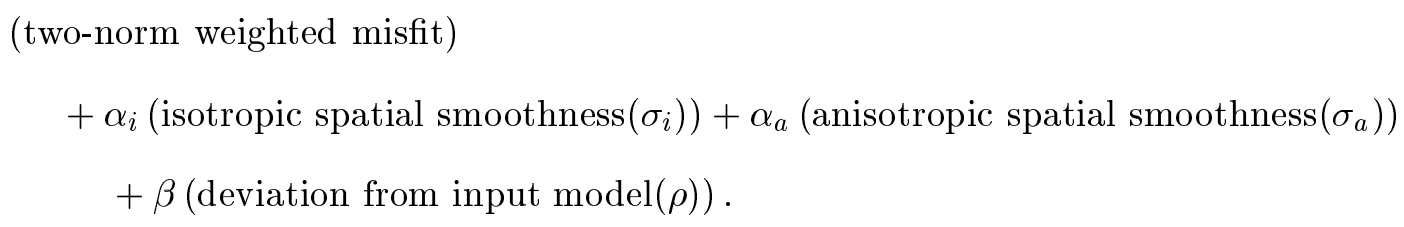

The weights in the misfit component of the penalty functional are the inverse of the measurement uncertainties. There are three trade-off parameters, $\alpha_{i}, \alpha_{a}$ and $\beta$ that weight the smoothness of the isotropic and anisotropic parts of the model and impose an explicit 
constraint on model norm in regions of poor data coverage. The smoothness constraint also depends on a spatial smoothing length or spatial correlation length parameter, $\sigma$, which is the standard deviation of the Gaussian spatial smoothing operator. A different correlation length is used for the isotropic $\left(\sigma_{i}\right)$ and anisotropic $\left(\sigma_{a}\right)$ parts of the model. The model norm constraint depends adaptively on the local path density, $\rho$. If the local path density is greater than about 50 paths for a given $2^{\circ} \times 2^{\circ}$ cell, the model-norm constraint vanishes. Except for small regions outside the Pacific at 150 sec period, path density is everywhere greater than this cut-off value so the model norm constraint is not applied. In practice, therefore, there are four "damping parameters" applied in the inversion: $\alpha_{i}$ and $\sigma_{i}$ that control the nature of the isotropic model and $\alpha_{a}$ and $\sigma_{a}$ that control the anisotropic part of the model. We typically refer to the $\alpha$ parameters as the "damping" parameters and the $\sigma$ parameters as the "smoothing" parameters, although in fact each parameter acts both to damp amplitudes and smooth the resulting maps. The effect of varying these parameters will be discussed in section 2.3 following.

The estimated model is defined relative to a radially anisotropic reference model, which at each period and wave-type is the dispersion map computed from the 3-D model of Shapiro and Ritzwoller (2002) that has been updated and described by Ritzwoller et al. (2004) for the Pacific. In each inversion, the isotropic, the $2 \psi$, and the $4 \psi$ coefficients are estimated simultaneously, but we will concentrate our discussion exclusively on the $2 \psi$ maps for Rayleigh waves. We define the amplitude of the $2 \psi$ component of azimuthal anisotropy at position $\mathbf{r}$ to be:

$$
A_{2 \psi}(\mathbf{r})=\frac{c_{A}^{\max }(\mathbf{r})-c_{r e f}(\mathbf{r})}{c_{r e f}(\mathbf{r})}=\frac{\sqrt{A_{1}^{2}(\mathbf{r})+A_{2}^{2}(\mathbf{r})}}{c_{r e f}(\mathbf{r})},
$$

where $A_{1}$ and $A_{2}$ are the $2 \psi$ anisotropy coefficients from equation (3), $c_{r e f}$ is the reference model speed, and $c_{A}^{\max }$ is the maximum speed with respect to $\psi$. The $4 \psi$ maps are included in the inversion to minimize the bias of the $2 \psi$ maps caused by their absence, and are damped similarly to the $2 \psi$ maps in the inversion.

The inversion procedure of Barmin et al. (2001) has been modified to include spatially extended surface wave sensitivity kernels for both minor arc (Ritzwoller et al., 2002) and major arc surface waves (Levshin et al., 2004). We refer to the use of these finite frequency kernels as "diffraction tomography", in contrast with "ray theory" which assumes that surface 
wave sensitivity is confined to an infinitesimally wide great-circle linking source and receiver. The inversions shown here are based on diffraction tomography for the isotropic part $\left(A_{0}\right)$, but ray theory for the anisotropic coefficients $\left(A_{1}, \ldots, A_{4}\right)$. We use ray theory for the anisotropic part of the model because the diffraction theory for anisotropy remains unclear to us. In particular, when using diffraction tomography to compute travel times through an azimuthally anisotropic model the local azimuth at the scatterer is not uniquely defined. By considering various alternative choices for the local azimuth, we argue in the Appendix that, at least at large scales across the Pacific, diffraction effects will not modify the characteristics of the anisotropic model that we interpret here. Ray theory for the anisotropic part of the model, therefore, will be sufficiently accurate for our purposes.

The inversion is global. The isotropic part of the model is represented on a $2^{\circ} \times 2^{\circ}$ spatial grid, but the anisotropic part of the model is on a $5^{\circ} \times 5^{\circ}$ grid.

\subsection{Choice of Damping and Smoothing}

The damping parameters $\alpha_{i}$ and $\alpha_{a}$ and the smoothing parameters $\sigma_{i}$ and $\sigma_{a}$ together determine the amplitude and smoothness of the perturbation from the input reference model. We show here that over a broad range of parameters the large-scale spatial pattern of azimuthal anisotropy across the Pacific is robust, but the amplitude of anisotropy is largely undetermined. The relative amplitude of azimuthal anisotropy as a function of lithospheric age, however, is fairly robust, but is not as well determined as the spatial pattern. All comments we make here about damping and smoothing hold for estimating azimuthal anisotropy only across the Pacific.

We find, but due to space limitations do not show here, that at large spatial scales such that $\sigma_{a}>10^{3} \mathrm{~km}$, the spatial pattern and relative amplitude of azimuthal anisotropy are robust relative to: data subsetting into two randomly selected equally sized data subsets, the use of ray theory versus diffraction theory for the isotropic part of the model, and changes across a broad range of isotropic damping $\left(\alpha_{i}\right)$ and smoothing $\left(\sigma_{i}\right)$ parameters. In particular, the pattern and relative amplitude of azimuthal anisotropy are robust even when we allow the isotropic part of the model to have unphysically large amplitudes and much short wavelength structure. Because the choice of the isotropic smoothing and damping parameters are of little 
consequence to the anisotropy results presented below, we fix $\sigma_{i}=300 \mathrm{~km}$ and the relative value of $\alpha_{i}=4 / 3$. (The values of $\alpha_{i}$ and $\alpha_{a}$ reported here are only relative values, defined such that $\alpha_{a} \sim 1$ in the middle of the band of "acceptable" damping parameters discussed below.) As shown in the Appendix, the spatial pattern and relative amplitude of azimuthal anisotropy are also robust at large-scales to the choice of the use of ray theory versus diffraction theory for the anisotropic part of the model.

The situation is more troublesome with respect to the choice of the anisotropy damping $\left(\alpha_{a}\right)$ and smoothing $\left(\sigma_{a}\right)$ parameters. If $\sigma_{a}$, the anisotropy correlation length, is much smaller than $\sim 10^{3} \mathrm{~km}$, the spatial pattern of anisotropy becomes unstable, being susceptible to relatively small changes in the other parameters in the inversion. Thus, only the large scale pattern of anisotropy can be resolved with confidence and we fix $\sigma_{a}=10^{3} \mathrm{~km}$ in all subsequent results.

The choice of the damping parameters in some inversions can be guided by fit to the data. Unfortunately, misfit is not a reliable guide to the choice of the anisotropy damping parameter as only relatively small improvements in fit to the data are afforded by changing $\alpha_{a}$. For example, as Figure 2 illustrates, reducing $\alpha_{a}$ by an order of magnitude from 10/3 to $1 / 3$ will change rms-misfit for the 50 sec Rayleigh wave group speed only from 16.0 sec to 15.3 sec. For comparison, the misfit for PREM (Dziewonski and Anderson, 1981) to the 50 sec Rayleigh wave group speed data is about $66 \mathrm{sec}$, so the maps produced with $\alpha_{a}$ equal to $10 / 3$ and $1 / 3$ generate variance reductions of about $94 \%$ and $94.5 \%$ relative to PREM, respectively. The isotropic part of the model alone produces a misfit of about $17 \mathrm{sec}(\sim 93 \%$ variance reduction relative to PREM) and the 3-D model of Shapiro and Ritzwoller (2002) generates a misfit of about $20 \mathrm{sec}(\sim 91 \%$ relative to PREM). A misfit value of about $15.3 \mathrm{sec}$ can be attained from a purely isotropic model by greatly reducing isotropic damping, but the amplitudes of the resulting models are so large and the patterns of the anomalies so unstable that the maps can be rejected on physical grounds.

Tighter constraints on the choice of the anisotropy damping parameter derive by considering the amplitude of anisotropy. Figure 3 shows that $\alpha_{a}$ can vary over a broad-range and for the 50 sec Rayleigh wave group speed will produce an average amplitude of anisotropy less than about $1 \%$ with peak amplitudes less than about $4 \%$. This range includes values of $\alpha_{a}$ 
as small as about $1 / 3$. As $\alpha_{a}$ increases above about 3 , the amplitude of azimuthal anisotropy becomes negligibly small. We, therefore, will restrict $\alpha_{a}$ to lie within the order of magnitude from $1 / 3$ to $10 / 3$. Similar assessments at different periods result in the same range of allowed values for $\alpha_{a}$. We seek inferences that are common to all maps with the anisotropic damping parameter $\alpha_{a}$ in this band.

\section{Results}

As discussed in section 2, we seek inferences that are common to all maps of azimuthal anisotropy in which the anisotropic smoothing parameter $\sigma_{a}=10^{3} \mathrm{~km}$ and the anisotropic damping parameter $\alpha_{a} \in[1 / 3,10 / 3]$. These parameters have been chosen to provide robustness relative to arbitrary choices of the damping parameters and to ensure that the peak amplitude of the resulting anisotropy maps are physically reasonable.

Figure 4 displays selected maps of azimuthal anisotropy from this range of anisotropic smoothing and damping parameters for the 50 sec Rayleigh wave group speed. There are prominent differences among these maps; in particular, as noted before (Fig. 3) the amplitude of anisotropy changes appreciably as a function of $\alpha_{a}$. There are, however, common features that we argue here are worthy of interpretation. In particular, the large-scale pattern of anisotropy across the Pacific is similar in each case. This can be illustrated by use of the "coherence function" $\mathrm{K}(\delta)$ introduced by Griot et al. (1998). This function is a measure of the spatial similarity between the patterns of azimuthal anisotropy in two maps and is defined as follows:

$$
K(\delta)=\frac{\sum_{\theta} \sum_{\phi} U_{1}(\theta, \phi) U_{2}(\theta, \phi) \sin (\theta) \exp \left(-\frac{\left(\psi_{\max , 1}(\theta, \phi)-\psi_{\max , 2}(\theta, \phi)+\delta\right)^{2}}{2 D_{\text {corr }}^{2}}\right)}{\left(\sum_{\theta} \sum_{\phi} \sin (\theta) U_{1}^{2}(\theta, \phi)\right)^{1 / 2}\left(\sum_{\theta} \sum_{\phi} \sin (\theta) U_{2}^{2}(\theta, \phi)\right)^{1 / 2}} .
$$

$U_{1}(\theta, \phi)$ and $U_{2}(\theta, \phi)$ are the amplitudes of the two maps at each point $(\theta, \phi)$ where $\theta$ is the co-latitude and $\phi$ is the longitude, where $U=\sqrt{A_{1}^{2}+A_{2}^{2}}$ with the anisotropy coefficients $A_{1}$ and $A_{2}$ defined by equation (3), and $\psi_{\max , 1}$ and $\psi_{\max , 2}$ are the azimuths at which the two wave speeds maximize. $D_{\text {corr }}$ is the uncertainty in the direction of anisotropy, which we set to $10^{\circ}$ to match the smoothing operator $\left(\sigma_{a}=10^{3} \mathrm{~km}\right)$, and determines the width of the coherence 
function. Equation (5) measures the correlation between two maps when one is rotated by angle $\delta$ relative to the other. Although anisotropy is estimated globally, we compute coherence only across the Pacific, setting anisotropy outside the Pacific to zero.

If two maps correlate perfectly, $\mathrm{K}(\delta)$ will appear as a narrow symmetric Gaussian-shaped function centered on $\delta=0$ with unit amplitude. An example is presented in Figure 5a, which is the coherence between two 50 sec Rayleigh wave group speed maps estimated with $\alpha_{a}$ of $1 / 3$ and 1, respectively. These are the two maps shown in Figures $4 \mathrm{a}$ and $4 \mathrm{~b}$. The coherence function is relatively narrow, peaks near to the origin, and is approximately symmetric about the origin. When the coherence function does not display multiple maxima, we can summarize the result by the value of the peak coherence, which in the example in Figure 5a is about 0.75. Peak coherence is displayed for a wide range of anisotropy damping parameters $\alpha_{a}$ in Figure 5b. For $\alpha_{a} \in[1 / 3,10 / 3]$, coherence is high, consistent with what can be ascertained by visual comparison within Figure 4, that the large-scale spatial pattern of anisotropy across the Pacific is robust. This result is only for the 50 sec Rayleigh wave group speed, but similar results are obtained at the other periods.

Although the absolute amplitude of azimuthal anisotropy is not robust relative to physically reasonable variations in the damping of anisotropy, $\alpha_{a}$, the relative amplitude of anisotropy across the Pacific is more stable, as Figure 6 illustrates. For the 50 sec Rayleigh wave group speed, the strength of anisotropy nearly halves on average from the ridge to a lithospheric age of about $125 \mathrm{Ma}$ and then flattens or slightly increases. Reducing $\alpha_{a}$ increases the variance within each age bin, but the trend remains in the bin means. At the weakest damping $\left(\alpha_{a} \sim 1 / 3\right)$, strong small-scale anisotropy is introduced in the far western Pacific east of the Philippine Plate that is below the resolution at larger values of the damping parameter.

Figure 7 shows maps of the $2 \psi$ component of azimuthal anisotropy for Rayleigh wave group speeds at four periods: $25 \mathrm{sec}, 50 \mathrm{sec}, 100 \mathrm{sec}$, and $150 \mathrm{sec}$. These maps derive from the value of $\alpha_{a}$ in the middle of the range of accepted values; i.e., $\alpha_{a}=1$. Amplitudes generally decrease with period, but it is difficult to compare amplitudes quantitatively across periods because absolute amplitudes are poorly constrained. The patterns of anisotropy at long-periods (100 sec, $150 \mathrm{sec}$ ) are very similar, but the shorter period maps (25 sec, $50 \mathrm{sec}$ ) disagree with the longer period maps, particularly in the western Pacific. This can be seen 
more clearly in the peak coherence values listed in Table 1. We will show below that long period fast-axis directions align with present-day plate motions across the entire Pacific. At shorter periods, however, they align well with present day plate motions only in the young Pacific.

Figure 8 displays Rayleigh wave group and phase speed sensitivity kernels to isotropic shear wave speed, which are similar to the sensitivity kernels for anisotropic shear wave speed for typical elastic tensors (Becker et al., 2003). The Rayleigh wave group speed sensitivity kernels peak at about $30 \mathrm{~km}, 50 \mathrm{~km}, 90 \mathrm{~km}$, and $140 \mathrm{~km}$ for $25 \mathrm{sec}, 50 \mathrm{sec}, 100 \mathrm{sec}$, and $150 \mathrm{sec}$ period, respectively. The phase sensitivity kernels peak deeper at each period $(40 \mathrm{~km}, 70 \mathrm{~km}$, $125 \mathrm{~km}$, and $200 \mathrm{~km}$ at $25 \mathrm{sec}, 50 \mathrm{sec}, 100 \mathrm{sec}$, and $150 \mathrm{sec}$ ) and are heavier-tailed with depth. The negative lobe on the group speed kernels, in particular, renders difficult direct comparison between our group speed maps and the phase speed maps published by other researchers, although the best comparison of group and phase speed maps would probably be between the 50 sec phase and $100 \mathrm{sec}$ group speed maps.

\section{Discussion}

\subsection{Comparison Between $2 \psi$ Fast Axis Directions and Present-Day Plate Motions}

Previous studies of the azimuthal anisotropy of Rayleigh wave phase speeds between about $40 \mathrm{sec}$ and $150 \mathrm{sec}$ period across the Pacific have noted that $2 \psi$ fast axis directions align approximately with present-day plate motions. This is particularly striking near the East Pacific Rise where fast axis directions are oriented nearly perpendicular to the ridge. We test this hypothesis with the Rayleigh wave group speed maps from 25 sec to 150 sec period

(Fig. 7) by comparing with the present-day plate motion model HS3-NUVEL-1A of Gripp and Gordon (2002) shown in Figure 9. As Figure 8 illustrates, the short period group velocities provide more sensitivity to anisotropy in the shallow uppermost mantle than the intermediate and long period phase speeds. The results of this comparison are shown in Figures 10 - 12 .

We map the difference in azimuth between the direction of present-day plate motion and the anisotropic fast axis direction for Rayleigh wave group speeds from $25 \mathrm{sec}$ to $150 \mathrm{sec}$ period in Figure 10. At long periods, particularly at $150 \mathrm{sec}$, the $2 \psi$ fast axis directions align with the present day plate directions across most of the Pacific. Notable exceptions occur mainly 
near discontinuities in the plate motion model where the seismic model cannot resolve rapid spatial changes; e.g., near the Tonga-Fiji arc and along the Pacific-Antarctic Ridge. As periods reduce below $150 \mathrm{sec}$, however, there is a systematic deviation from present-day plate motions. Figure 11 demonstrates this with normalized weighted histograms of the azimuth differences. The weighted azimuth differences are also plotted versus lithospheric age in Figure 12. At 150 sec, although there is a slight trend with increasing lithospheric age, the anisotropic fast axes lie within about $20^{\circ}$ of current plate directions irrespective of the age of the lithosphere. At shorter periods, the discrepancy with lithospheric age increases. Note, however, that even at

25 sec period the anisotropic fast axis directions, on average, lie within $20^{\circ}-25^{\circ}$ of current plate motion directions in the young Pacific. At $25 \mathrm{sec}$ period it is clear that the deviation grows most rapidly after an age between 40 and $70 \mathrm{Ma}$. A similar on-set time for the deviation of the direction of anisotropy from present plate motion appears to be present but is more subtle at longer periods.

In conclusion, although at all periods group velocity anisotropic fast axis directions are approximately aligned with present day plate motions in the young Pacific, only the very long period group speed maps have fast axis directions oriented with current plate motions in the old Pacific. At shorter periods, which are more sensitive to the shallow lithosphere, fast axis directions in the old Pacific are significantly different from the present plate directions.

\subsection{Comparison Between $2 \psi$ Fast Axis Directions and Paleo-Spreading Directions}

If the anisotropic directions at short periods are not oriented along the direction of current plate motions in the old Pacific, what does or has controlled their orientation? It turns out that the anisotropic fast axis directions for the shorter period maps align more closely with the direction the plate was moving at the time of its formation.

To estimate this direction we compute the "paleo-spreading direction" from the gradient of lithospheric age. The direction of the local gradient of the lithospheric age $A$ will coincide with the paleo-spreading direction everywhere but near age boundaries, such as transform faults or the mid-oceanic ridge where the direction of the local age gradient is unstable even though its magnitude can be very large. Therefore, to estimate an average paleo-spreading direction in a particular region, the points located in the vicinity of plate boundaries must 
be excluded from the analysis. As a practical matter, we estimated the regional average paleo-spreading directions using the following two-step procedure. First, we computed the local age gradient $\nabla A(\mathbf{r})$ at each point $\mathbf{r}$ on a $0.1^{\circ}$ grid from the compilation of lithospheric age by Mueller et al. (1997). In the second step, we used the gradient to compute the weighted two-dimensional covariance matrix in a moving two-dimensional Gaussian window $G(\mathbf{r})$ :

$$
C_{x y}(\mathbf{r})=\int_{S} \nabla_{x} A\left(\mathbf{r}^{\prime}\right) \nabla_{y} A\left(\mathbf{r}^{\prime}\right) G\left(\left|\mathbf{r}-\mathbf{r}^{\prime}\right|\right) w\left(\mathbf{r}^{\prime}\right) d \mathbf{r}^{2}
$$

where $\sigma=1500 \mathrm{~km}$ in the Gaussian window (close to the lateral resolution for azimuthal anisotropy), the subscripts $x, y$ denote the $x$ and $y$ components of the gradient vector and the covariance matrix, and the weight $w\left(\mathbf{r}^{\prime}\right)$ approaches zero when the amplitude of the age gradient is either very large (e.g., near transform faults) or very small (i.e., if the age gradient is poorly defined). The regional average paleo-spreading direction is defined as the direction of the eigenvector of the matrix $\mathbf{C}$ corresponding to its larger eigenvalue. We refer to this as a spreading direction rather than plate motion because it only represents the component of plate motion perpendicular to the ridge. The component of plate motion parallel to the ridge is not revealed by taking the gradient of the age.

The final estimate of the local paleo-spreading directions is shown in Figure 13. Comparison with present day plate motion in Figure 9 shows that the paleo-spreading directions are similar to plate motion in the young Pacific, but deviate significantly in the old Pacific. More detailed inspection reveals that the paleo-spreading directions point approximately parallel to fracture zones which curve away from the direction of current plate motion in the old Pacific.

The results of the comparison between the anisotropic fast-axis directions and the paleo-spreading directions are shown in Figures 14 - 16 and should be contrasted with the comparison between the fast-axis directions and present plate motion directions seen in Figures 10 - 12. The contrast is that the worst agreement is at $150 \mathrm{sec}$ period and agreement between the fast-axis directions and the paleo-spreading directions is best at shorter periods, particularly at $50 \mathrm{sec}$. Comparison of Figures $16 \mathrm{~b}$ and $16 \mathrm{~d}$ is telling. At 50 sec period, the agreement between the fast-axis directions and the paleo-spreading directions is nearly independent of lithospheric age, but the discrepancy grows strongly with age at 150 sec period. As periods decreases below $50 \mathrm{sec}$, there is increasing sensitivity to anisotropy in the very 
shallow lithosphere where anisotropy may reflect processes of crustal formation and differ from that in the deeper lithosphere and asthenosphere. This may be why the maps at 25 sec (Fig. 14a) and below disagree with the paleo-spreading direction more than the intermediate period maps such as the one shown at 50 sec period (Fig. 14b).

\subsection{Two-Layered Stratification of Azimuthal Anisotropy in the Uppermost Mantle}

The results in sections 4.1 and 4.2 indicate that in the shallow lithosphere, anisotropic fast axis directions tend to align with the paleo-spreading direction. In the deeper lithosphere and asthenosphere, the anisotropic fast axes are oriented more nearly along present day plate motions. We infer from this, therefore, that anisotropy in the cool uppermost mantle is controlled by conditions that prevailed at the time of formation of the lithosphere. Subsequent changes in mantle flow are not revealed by anisotropy in the shallowest mantle. In the warmer asthenosphere, however, anisotropy has re-oriented to conform to current conditions of flow that are revealed by present-day plate motion. This "two-layer" model of mantle anisotropy beneath the Pacific is presented in idealized form in Figure 17. Anisotropic fast axes are oriented parallel to current mantle flow in the deep lithosphere and asthenosphere, but are "frozen" or fixed in the lithosphere revealing the strain conditions active during the early evolution of the lithosphere.

An idea of the depth extent of the layer of fixed anisotropy can be obtained from Figure 18 in which the $2 \psi$ azimuthal anisotropy maps on a grid of periods ranging from 20 to 150 sec were used to illustrate the period dependence of the azimuthal difference between the anisotropic fast axis and the plate motion directions along a great circle path from the Western Pacific to the East Pacific Rise. Because the depths sampled by surface waves increase with

period, as shown in Figure 8, period can be used an an approximate proxy for depth. Figure 18 illustrates that large differences between the fast axis of azimuthal anisotropy and the direction of present-day plate motion set-on between $\sim 40-70 \mathrm{Ma}$ and exist primarily at periods below about 80 sec. Because the sensitivity of 80 sec Rayleigh group speed peaks at about $80 \mathrm{~km}$ depth, the zone of fixed anisotropy probably does not extend much deeper than about $100 \mathrm{~km}$ in the Western Pacific. This depth of the transition from fixed to conforming anisotropy in the old Pacific is, therefore, probably somewhat deeper than the depth of the 
hypothesized " $G$-discontinuity", a compositional boundary that separates the lithosphere from the asthenosphere as discussed by Gaherty et al. (1996, 1999). As Figure 18 shows, the depth of the transition from the fixed to the conforming layer also appears to increase with age. This depth does, however, vary regionally, and a quantitative discussion of its average characteristics, trend with age, and regional variability is beyond the scope of the present work.

Another line of evidence supporting a two-layer model of anisotropy in the upper mantle beneath the Pacific results from a consideration of how the amplitude of anisotropy (eq. (4)) varies with period and lithospheric age. Figure 19 shows that at long periods there is a decrease in amplitude in the old Pacific relative to the amplitudes in the young Pacific, as first documented by Nishimura and Forsyth $(1988,1989)$. The amplitude of anisotropy on the $25 \mathrm{sec}$ map does not decrease in the old, western Pacific and the biggest old-young discrepancy in amplitude comes at $100 \mathrm{sec}$ period. The old-young (west-east) discrepancy can result from destructive interference at periods sensitive to anisotropy in both layers. Thus, at $25 \mathrm{sec}$ period where sensitivity is exclusively limited to the layer in which anisotropy is frozen into the lithosphere, the strength of anisotropy does not decrease in the old Pacific. Similarly, at $150 \mathrm{sec}$ which is dominantly (although not exclusively) sensitive to the deeper layer of anisotropy, the decrease in the strength of anisotropy with age will be weaker than at the intermediate periods of $50 \mathrm{sec}$ and $100 \mathrm{sec}$. At $50 \mathrm{sec}$ and $100 \mathrm{sec}$ period, the old-young (west-east) discrepancy is largest because the waves are sensitive both to the fixed anisotropy of the upper lithosphere and the conformable anisotropy of the underlying asthenosphere.

This argument to explain the trend of the amplitude of azimuthal anisotropy with lithospheric age is purely qualitative and is intended only as a consistency check of the two-layer model of azimuthal anisotropy. There may be other plausible sources for this decrease in azimuthal anisotropy in the deep lithosphere to upper asthenosphere in the west Pacific. For example, the hypothesized change in the direction of plate motion at about 43 Ma (e.g., Lithgow-Bertelloni and Richards, 1998) could cause a decrease in the amplitude of anisotropy in the asthenosphere and lower lithosphere, where some fraction of the olivine crystals may have kept their original orientation after the plate changed directions. This potential change in plate motion, however, is contentious (e.g., Norton, 1994; Tarduno et al., 
2003). The decrease in the amplitude of azimuthal anisotropy could also be evidence for a disruption in the deep lithosphere or asthenosphere of the central Pacific that could arise from thermal boundary layer instabilities (e.g., Ritzwoller et al., 2004) or multiple thermal plumes, but modeling needs to be performed in order to explain simultaneously the amplitude pattern, the fast axis directions, and the nature of the isotropic part of the model.

\subsection{Potential Cause(s) of the Stratification of Anisotropy}

There are several alternative processes that could produce the two layered stratification of anisotropy across the Pacific upper mantle. We mention two hypotheses that we believe to be the most prominent alternatives. The first hypothesis is that the stratification of anisotropy is thermally generated, being predominantly controlled by mantle temperature and the thermal history of the lithosphere. Within this category there are two sub-classes of processes that may produce the stratification. The first process is that the stratification of anisotropy is controlled directly by mantle rheology; lower temperatures in the shallow lithosphere prohibit dislocation creep from occurring as fast as in the asthenosphere. Under this interpretation, anisotropy would be formed in the shallow lithosphere during the early history of the plate, but because lithospheric temperatures are too cool for dislocation creep to reorient crystal axes as mantle flows change, the orientation of anisotropy in the lithosphere would remain roughly fixed. In the warmer underlying asthenosphere, however, the warmer temperatures and lower viscosity permit crystallographic reorientation to conform with changes in mantle flows. The second process is also controlled by temperature but goes farther by specifying the mechanism of the formation of anisotropy. In fact, it is the consequence of the first process. It asserts that anisotropy will form only near shear flows and, as the lithosphere grows, the upper reaches of shear flow will deepen into the mantle. Under this interpretation (e.g., Podelefsky et al., 2004), anisotropy will be frozen into the shallow lithosphere because the shear strains that can reorient crystallographic axes will occur at increasing depths as the plate ages. Both of these processes can qualitatively explain the general characteristics of the observations that anisotropy is able to conform with current flow conditions in the asthenosphere and the deep lithosphere and that anisotropy in the shallow lithosphere appears to be "frozen in" and aligns nearer to the paleo-spreading direction. It remains to be determined if these processes will 
be able quantitatively to reproduce the average characteristics of the observations. Whether these processes will predict that the transition depth from frozen to conforming anisotropy will deepen with plate age (e.g., Fig 18) will bear on the nature of the temporal changes in the mantle flow regime. If, for example, the direction of plate motion has changed only once during the history of the Pacific lithosphere and this reflects the dominant change in mantle flow, then one would expect that the transition depth would be approximately constant in the old Pacific. If mantle flows change more often, then a more complicated spatial pattern of the interface between fixed and conforming anisotropy would emerge.

A second hypothesis is that the stratification of anisotropy is controlled by upper mantle composition and water content, in particular by vertical compositional heterogeneity remnant from melt separation that occurred during plate formation. Under this interpretation, the upper lithosphere will be depleted in water relative to the underlying asthenosphere (e.g., Gaherty et al., 1996, 1999) and this will strongly affect mantle rheology (Hirth and Kohlstedt, 1996) and, hence, the ability of anisotropy to reorient in response to changing mantle flows as well as its relation to mantle strain (Jung and Karato, 2001). This hypothesis, in principle, can explain the existence of anisotropy stratification, in which anisotropy is fixed in the upper layer and conforms to the ambient flow regime in the lower layer. There are, however, several qualitative challenges for this hypothesis. First, the G-discontinuity which Gaherty et al. $(1996,1999)$ have hypothesized to mark the transition from low to high water content may be shallower $(\sim 70 \mathrm{~km})$ than the depth of the transition from fixed to conforming anisotropy in the upper mantle in the Western Pacific (closer to $100 \mathrm{~km}$ ). The observation of the transition depth requires further clarification, however. Second, it may be difficult for this hypothesis to generate significant topography on the interface between fixed and conforming anisotropy. The observation of the deepening of the transition region with age, however, also requires further work to document and clarify. Finally, Jung and Karato (2001) argue that when large amounts of water at high stresses are introduced into olivine, the relation between the flow direction and the induced anisotropy changes. Our findings suggest that at large-scales across the Pacific, the relation between anisotropy and flow direction (present or at the time of lithospheric formation, depending on the depth) is, on average, rather simple. In fact, the relation between current plate motions in the hot-spot frame and anisotropic fast axes in the 
hypothetically wet asthenosphere are particularly in good agreement. If the experiments of Jung and Karato (2001) are relevant to asthenospheric conditions, this would argue against significant water content in the asthenosphere across much of the Pacific and would undercut the hypothesis that stratification of water content controls the stratification of anisotropy. This would not preclude the possibility that large amounts of water are locally present in the upper mantle, for example due to enrichment of volatile content near subduction zones.

Given the state of the observations presented here, it is impossibly to differentiate definitively between the two competing hypotheses that anisotropy stratification is controlled by composition or thermal processes. We have, however, identified observables that may provide the critical tests; namely, the depth of the transition from the fixed to the conforming layers and the spatial variation of this depth, particularly with lithospheric age. Current observations may favor the thermal hypothesis, but more work is needed to clarify the critical observables and perform modeling experiments to fit the observations quantitatively.

\section{Appendix: On Diffraction Tomography in Anisotropic Media}

Surface wave "diffraction tomography" (e.g., Ritzwoller et al., 2002) is based on a Born-approximation solution to the two-dimensional acoustic wave equation (e.g., Spetzler and Snieder, 2001) that provides a reasonable approximation for the propagation of a single-mode surface wave (Rayleigh or Love) in a slightly heterogeneous, isotropic medium. Within this approximation, travel time perturbations are estimated as surface integrals with two-dimensional sensitivity kernels, in contrast with the line integrals along rays that emerge in traditional ray theory. Finite frequency effects should also be accounted for when using surface-wave tomography to estimate travel times through an azimuthally anisotropic medium. We are not aware, however, of the existence of a theory that describes the anisotropic finite-frequency surface-wave sensitivity kernels. In the absence of a solid theoretical foundation, we introduce anisotropic kernels by simple analogy with the isotropic theory. At the center of the analogy, however, is an ambiguity concerning the definition of the angle at the scatterer. We consider several alternative definitions for this angle and show that, irrespective of this choice, the principal features of azimuthal anisotropy estimated across the Pacific remain relatively unchanged. 


\section{Travel Time Through an Anisotropic Medium: Ray theory}

Ray-theoretical travel time perturbations in an anisotropic medium can be written as an integral along the ray path $p$ (e.g., Barmin et al, 2001):

$$
\delta t^{r}=\int_{p} \frac{m(\mathbf{r})}{c_{0}} d s
$$

where $c_{0}$ is the reference phase or group speed and:

$$
\begin{aligned}
& m(\mathbf{r})=\sum_{k=0}^{4} \gamma_{k}(\psi(\mathbf{r})) m_{k}(\mathbf{r}) \\
& \gamma_{0}(\psi)=1 \quad m_{0}(\mathbf{r})=\left(c_{0}-c_{I}(\mathbf{r})\right) / c_{I}(\mathbf{r}) \\
& \gamma_{1}(\psi)=-\cos (2 \psi) \quad m_{1}(\mathbf{r})=A_{1}(\mathbf{r}) c_{0}(\mathbf{r}) / c_{I}^{2}(\mathbf{r}) \\
& \gamma_{2}(\psi)=-\sin (2 \psi) \quad m_{1}(\mathbf{r})=A_{2}(\mathbf{r}) c_{0}(\mathbf{r}) / c_{I}^{2}(\mathbf{r}) \\
& \gamma_{3}(\psi)=-\cos (4 \psi) \quad m_{1}(\mathbf{r})=A_{3}(\mathbf{r}) c_{0}(\mathbf{r}) / c_{I}^{2}(\mathbf{r}) \\
& \gamma_{4}(\psi)=-\sin (4 \psi) \quad m_{1}(\mathbf{r})=A_{4}(\mathbf{r}) c_{0}(\mathbf{r}) / c_{I}^{2}(\mathbf{r})
\end{aligned}
$$

using notation from equations (1)-(3). The $k=0$ term corresponds to isotropic perturbations and the $k=1, \ldots, 4$ terms describe the $2 \psi$ and the $4 \psi$ anisotropic effects. For convenience, we can rewrite the travel time perturbation as a sum of contributions from the isotropic and anisotropic parts:

$$
\begin{gathered}
\delta t^{r}=\delta t_{I}^{r}+\delta t_{A}^{r} \\
\delta t_{I}^{r}=\int_{p} \frac{c_{0}-c_{I}(\mathbf{r})}{c_{0} c_{I}(\mathbf{r})} d s \\
\delta t_{A}^{r}=\sum_{k=1}^{4} \int_{p} \frac{\gamma_{k}(\psi(\mathbf{r})) m_{k}(\mathbf{r})}{c_{0}} d s .
\end{gathered}
$$

\section{Travel Time Through an Isotropic Medium: Diffraction Theory}

In an isotropic medium, equation (A4) is reduced to equation (A5). In this case, the transition from ray theory to diffraction theory is achieved by substituting the linear integral along the path with a surface integral:

$$
\delta t_{I}^{d}=\int_{S} K(\mathbf{r}) \frac{c_{0}-c_{I}(\mathbf{r})}{c_{0} c_{I}(\mathbf{r})} d \mathbf{r}^{2}
$$


where $K(\mathbf{r})$ is the diffraction sensitivity kernel (e.g., Spetzler and Snieder, 2001; Ritzwoller et al., 2002) and $d \mathbf{r}^{2}$ is an area-element.

\section{Travel Time Through an Anisotropic Medium: Diffraction Theory}

To write diffraction-theoretic travel times in an anisotropic medium analogous to the isotropic case we replace the linear integrals along the ray with the surface integrals:

$$
\delta t_{A}^{d}=\sum_{k=1}^{4} \int_{S} K(\mathbf{r}) \frac{\gamma_{k}(\psi(\mathbf{r})) m_{k}(\mathbf{r})}{c_{0}} d \mathbf{r}^{2},
$$

where $K(\mathbf{r})$ is the same kernel as in the isotropic case. The principal problem with this ad-hoc definition of the anisotropic diffraction kernels is that the local propagation angle at the scatterer, $\psi(\mathbf{r})$, and, consequently, the anisotropic kernels are not uniquely defined outside the great-circle connecting the source and the receiver. There are several alternative ways to define the angle at the scatterer, some of which are shown in Figure 20. The use of the angle $\psi_{1}$ (the direction of the scatterer from the source) or the angle $\psi_{2}$ (the direction of the scatterer from the receiver) has the disadvantage that reciprocity is not preserved. The use of angle $\psi_{3}$, the average of $\psi_{1}$ and $\psi_{2}$, will satisfy reciprocity as would the angle parallel to the great-circle (not shown in Fig. 20). We have considered three versions of the diffraction theory through an anisotropic medium based on sensitivity kernels that use angles $\psi_{1}, \psi_{2}$, and $\psi_{3}$.

The respective kernels, $K_{A}^{d 1}(\mathbf{r}), K_{A}^{d 2}(\mathbf{r})$, and $K_{A}^{d 3}(\mathbf{r})$, will generate three different travel times through the same azimuthally anisotropic model: $\delta t_{A}^{d 1}, \delta t_{A}^{d 2}$, and $\delta t_{A}^{d 3}$. We note that these three angles differ from each other most appreciably away from the middle of the Fresnel zone; i.e., near the source and receiver. Therefore, the theories deviate most and are most uncertain near sources and receivers.

\section{Azimuthal Anisotropy Estimated with Diffraction Theory and Ray Theory}

We assume that none of these theories governing the effect of diffraction through an anisotropic medium is right, but attempt to use them to quantify the expected magnitude of the effect of diffraction on surface waves propagating in an anisotropic medium. In all of the inversions, we used diffraction theory for the isotropic part of the model but different theories (ray and diffraction) for the anisotropic part of the model. The ray theoretic results, therefore, refer to the use of diffraction theory for the isotropic part of the model and ray theory for the 
anisotropic part. The diffraction theoretic results use standard diffraction theory for the isotropic part (Ritzwoller et al., 2002) but one of the three variants of diffraction theory for the anisotropic part of the model.

We find, first, that the azimuthal anisotropy maps estimated with the diffraction theories based on kernels $K_{A}^{d 1}(\mathbf{r}), K_{A}^{d 2}(\mathbf{r})$, and $K_{A}^{d 3}(\mathbf{r})$ are more similar to one another than any of them are to the anisotropy estimated using ray theory. The effect of incorporating diffraction, therefore, can be illustrated with the anisotropy maps that emerge from just one of the three variants of the theory. Second, Figure 21 shows that for the 50 sec Rayleigh wave group speed with our choice of smoothing and damping parameters, the large-scale pattern of $2 \psi$ azimuthal anisotropy across Pacific is not strongly different from that which emerges from ray theory. The exception occurs in the Central Pacific (Fig. 21c) where the amplitude of anisotropy is small. There is, however, a much more significant difference in continents. There are similar results at other periods. Finally, the difference between the theories is also more prominent if we damp the anisotropic maps less.

The results shown in Figure 21 can be partially understood in light of the fact that the wave paths across most of the Pacific tend to be long. The biggest differences among the diffraction theories themselves and between them and ray theory occur near sources and receivers, which are relatively rare across the Pacific. It is near source locations (i.e., plate boundaries generally), near receiver locations (i.e., continents generally), and for smaller scale structures that the effects of diffraction through the anisotropic medium become important. This is a cautionary note for the estimation of azimuthal anisotropy in continental areas, in particular, but across the Pacific the large-scale results are robust relative to the theory chosen.

\section{Acknowledgement}

This work benefited from discussions with Thorsten Becker, Martha Savage, Roel Snieder, and Shi-Jie Zhong as well as careful reviews by Thorsten Becker, Donald Forsyth, and James Gaherty. We gratefully acknowledge the staffs at the IRIS-DMC and the GEOFON and GEOSCOPE data centers for providing most of the waveform data on which results are based. As always, we are grateful to Mikhail Barmin for assistance with the surface wave tomography. 
All maps were generated with the Generic Mapping Tools (GMT) data processing and display package (Wessel and Smith, 1991, 1995). This work was supported by grants from the US National Science Foundation (OPP-0125848, OPP-0136103, EAR-0409217). 


\section{References}

Aki, K. and K. Kaminuma, Phase velocities in Japan. Part I. Love waves from the Aleutian shock of March 9, 1957, Bull. Earthq. Res. Inst., 41, 243-259, 1963.

Babuska, V. \& Cara, M., Seismic Anisotropy in the Earth, Kluwer, Dordrecht, 1991.

Barmin, M.P., Levshin, A.L., \& Ritzwoller, M.H., A fast and reliable method for surface wave tomography, Pure appl. Geophys., 158, 1351-1375, 2001.

Becker, T.W., Kellogg, J.B., Ekström, G., \& O’Connell, R.J., Comparison of azimuthal seismic anisotropy from surface waves and finite-strain from global mantle-circulation models, Geophys. J. Int., 155, 696-714, 2003.

Blackman, D.K. and J.-M. Kendall, Seismic anisotropy of the upper mantle: 2. Predictions for current plate boundary flow models, Geochemistry, Geophysics, and Geosystems, 3 (2001GC000247), 2002.

Blackman, D.K. H.-R. Wenk, and J.-M. Kendall, Seismic anisotropy of the upper mantle: 1. Factors that affect mineral texture and effective elastic properties, Geochemistry, Geophysics, and Geosystems, 3 (2001GC000248), 2002.

Buttles, J. and P. Olson, A laboratory model of subduction zone anisotropy,Earth Planet. Sci. Lett., 164, 245-262, 1998.

Chastel, Y.B., P.R. Dawson, H.-R. Wenk, and K. Bennett, Anisotropic convection with implications for the upper mantle, J. Geophys. Res., 98, 17757-17771, 1993.

Dziewonski, A.M. and D.L. Anderson, Preliminary Reference Earth Model, Phys. Earth Planet. Inter., 25, 297-356, 1981.

Ekström, G., Mapping the lithosphere and asthenosphere with surface waves: Lateral structure and anisotropy, in M.A. Richards, R. Gordon, and R. van der Hilst (eds.), The History of Global Plate Motions, Am. Geophys. Un., Washington, D.C., 211-238, 2000.

Ekström, G. and A.M Dziewonski, The unique anisotropy of the Pacific mantle, Nature, 394, 168-172, 1998. 
Forsyth, D.W., The early structural evolution and anisotropy of the oceanic upper mantle, Geophys. J. R. Astr. Soc., 43, 103-162, 1975.

Gaherty, J.B., T.H. Jordan, and L.S. Gee, Seismic structure of the upper mantle in the central Pacific corridor, J. Geophys. Res., 101, 22,291-22,309, 1996.

Gaherty, J.B., M. Kato, and T.H. Jordan, Seismological structure of the upper mantle: a regional comparison of seismic layering, Phys. Earth Planet. Int., 110, 21-41, 1999.

Gaboret, C., A.M. Forte, and J.-P. Montagner, The unique dynamics of the Pacific hemisphere mantle and its signature on seismic anisotropy, Earth Planet. Sci. Lett., 208, 219-233, 2003.

Griot, D.A., Montagner, J.-P., and Tapponier, P., Surface wave phase velocity tomography and azimuthal anisotropy in Central Asia, J. geophys. Res., 103, 21215-21232, 1998.

Gripp, A.E. \& Gordon, R.G., Young tracks of hotspots and current plate velocities, Geophys. J. Int., 150, 321-361, 2002.

Hall, C.E., K.M. Fischer, and E.M Parmentier, The influence of plate motions on threedimensional back arc mantle flow and shear wave splitting, J. Geophys. Res., 105, 28009-28033, 2000.

Hess, H.H., Seismic anisotropy of the uppermost mantle under oceans, Nature, 203, 629-631, 1964 .

Hirth, G. and D.L. Kohlstedt, Water in the oceanic upper mantle: implications for rheology, melt extraction and the evolution of the lithosphere, Earth Planet. Sci. Lett., 144, 93-108, 1996.

Jung, H. and S. Karato, Water-induced fabric transitions in olivine, Science, 293, 1460-1463, 2001.

Kaminski, E. and N. Ribe, A kinematic model for the recrystallization and texture development in olivine polycrystals, Earth Planet. Sci. Lett., 189, 253-267, 2001. 
Larsen, E.W.F, J. Tromp, and G. Ekström, Effects of slight anisotropy on surface waves, Geophys. J. Int., 132, 654-666, 1998.

Levin, V., W. Menke, and J. Park, Shear wave splitting in the Appalachians and the Urals: A case for multilayer anisotropy, J. Geophys. Res., 104, 17975-17993, 1999.

Levshin, A.L., M.P. Barmin, M.H. Ritzwoller, and J. Trampert, Using both minor and major arc data for global surface wave diffraction tomography, submitted to Phys. Earth Planet. Ints., 2004.

Lithgow-Bertelloni, C., and M.A. Richards, The dynamics of Cenozoic and Mesozoic plate motions, Revs. Geophys., 36, 27-78, 1998.

McEvilly, T.V., Central US crust - upper mantle structure from Love and Rayleigh wave phase velocity inversion, Bull. Seism. Soc. Am., 54, 1997-2015, 1964.

McKenzie, D.P., Finite deformations during fluid flow, Geophys. J. R. Astr. Soc., 58, 689-715, 1979.

Montagner, J.-P., Where can seismic anisotropy be detected in the Earth's mantle? In boundary layers ..., Pure appl. Geophys., 151, 223-256, 1998.

Montagner, J.-P. and T. Tanimoto, Global upper mantle tomography of seismic velocities and anisotropies, J. geophys. Res., 96, 20337-20351, 1991.

Morris, G.B., R.W. Raitt, and G.G. Shor, Velocity anisotropy and delay-time maps of the mantle near Hawaii, J. Geophys. Res., 74, 4300-4316, 1969.

Mueller, R.D., Roest, W.R., Royer, J.-Y., Gahagan, L.M., \& Sclater, J.G., Digital isochrons of the world's ocean floor, J. Geophys. Res., 102, 3211-3214, 1997.

Nataf, H.-C., I. Nakanishi, and D.L. Anderson, Anisotropy and shear velocity heterogeneity in the upper mantle, Geophys. Res. Lett., 11, 109-112, 1984.

Nishimura, C.E. and D.W. Forsyth, Rayleigh wave phase velocities in the Pacific with implications for azimuthal anisotropy and lateral heterogeneities, Geophys. J., 94, 479-501, 1988. 
Nishimura, C.E. and D.W. Forsyth, The anisotropic structure in the upper mantle of the Pacific, Geophys. J., 96, 203-229, 1989.

Norton, I.O., Plate motions in the North Pacific: The 43 Ma non-event, Tectonics, 14, 1080-1094, 1995.

Peyton, V., V. Levin, J. Park, M. Brandon, J. Lees, E. Gordeev, and A. Ozerov, Mantle flow at a slab edge: Seismic anisotropy in the Kamchatka region, Geophys. Res. Lett., 28(2), 379-382, 2001.

Podelefsky, N.S., S. Zhong, and A.K. McNamara, The anisotropic and rheological structure of the oceanic upper mantle from a simple model of plate shear, Geophys. J. Int., 158, 287-296, 2004.

Raitt, R.W., G.G. Shor, T.J.G. Francis, and G.B. Morris, Anisotropy of the Pacific upper mantle, J. Geophys. Res., 74, 3095-3109, 1969.

Regan, J. and D.L. Anderson, Anisotropic models of the upper mantle, Phys. Earth Planet. Inter., 35, 227-263, 1984.

Ribe, N.M., Seismic anisotropy and mantle flow, J. Geophys. Res., 94, 4213-4223, 1989.

Ribe, N.M., On the relation between seismic anisotropy and finite strain, J. Geophys. Res., $97,8737-8747,1992$.

Ribe, N.M. and Y. Yu, A theory for plastic-deformation and textural evolution of olivine polycrystals, J. Geophys. Res., 96, 8325-8335, 1991.

Ritzwoller, M.H. \& Levshin, A.L., Eurasian surface wave tomography: group velocities, Geophys. J. Int., 134, 315-328, 1998.

Ritzwoller, M.H., Shapiro, N.M., Barmin, M.P., \& Levshin, A.L., Global surface wave diffraction tomography, J. geophys. Res., 107(B12), 2335, doi:10.1029/2002JB001777. 2002 .

Ritzwoller, M.H., N.M. Shapiro, and S. Zhong, Cooling history of the Pacific lithosphere, Earth Planet. Sci. Lett., in press, 2004. 
Romanowicz, B. and R. Snieder, A new formalism for the effect of lateral heterogeneity on normal modes and surface waves, II. General anisotropic perturbations, Geophys. J. R. Astr. Soc., 93, 91-99, 1988.

Russo, R.M. and P.G. Silver, Trench-parallel flow beneath the Nazca plate from seismic anisotropy, Science, 263, 1105-1111, 1994.

Savage, M.K., Seismic anisotropy and mantle deformation: What have we learned from shear wave splitting?, Rev. Geophys., 37, 65-106, 1999.

Savage, M.K. and P.G. Silver, The interpretation of shear-wave splitting parameters in the presence of two anisotropic layers, Geophys. J. Int., 119, 949-963, 1994.

Shapiro, N.M. \& Ritzwoller, Monte-Carlo inversion for a global shear velocity model of the crust and upper mantle, Geophys. J. Int., 151, 88-105, 2002.

Shapiro, N.M., M.H. Ritzwoller, P. Molnar, and V. Levin, Thinning and flow of Tibetan crust constrained by seismic anisotropy. Science, in press, 2004.

Shearer, P.M. and J.A. Orcutt, Compressional and shear wave anisotropy in the oceanic lithosphere - the Ngendei seismic refraction experiment, Geophys. J.R. Astr. Soc., 87, 967-1003, 1986.

Silver, P.G. and W.E. Holt, The mantle flow field beneath western North America, Science, 295, 1054-1057, 2002.

Smith, M.L. \& Dahlen, F.A., The azimuthal dependence of Love and Rayleigh waves propagation in a slightly anisotropic medium, J. geophys. Res., 78, 3321-3333, 1973.

Smith, M.L. \& Dahlen, F.A., Correction to 'The azimuthal dependence of Love and Rayleigh waves propagation in a slightly anisotropic medium', J. geophys. Res., 80, 1923, 1975.

Spetzler, J. and R. Snieder, The effects of small-scale heterogeneity on the arrival time of waves, Geophys. J. Int., 145, 786-796, 2001.

Tanimoto, T. and D.L. Anderson, Mapping convection in the mantle, Geophys. Res. Lett., $11,287-290,1984$ 
Tarduno, J.A., R.A. Duncan, D.W. Scholl, R.D. Cottrell, B. Steinberger, T. Thordarson, B.C. Kerr, C.R. Neal, F.A. Frey, M. Torii, C. Carvallo, The Emperor Seamounts: Southward motion of the Hawaiian hotspot plume in earth's mantle, Science, 301, 1064-1069, 2003

Tommasi, A., Forward modeling of the development of seismic anisotropy in the upper mantle, Earth Planet. Sci. Lett., 160, 1-13, 1998.

Trampert, J. \& Woodhouse, J.H., Global anisotropic phase velocity maps for fundamental mode surface waves between 40 and 150 s, Geophys. J. Int., 154, 154-165, 2003.

Vinnik, L., D. Peregoudov, L. Makeyeva, S. Oreshin, and S. Roecker, Towards 3-D fabric in the continental lithosphere and asthenosphere: The Tien Shan, Geophys. Res. Lett., 29(16), Art. No. 1791, 2002.

Wessel, P and W. H. F. Smith, Free software helps map and display data, Eos Trans. Amer. Geophys. Un., 72, 441, 1991.

Wessel, P., and W.H.F. Smith, New version of the Generic Mapping Tools released, Eos Trans. Amer. Geophys. Un., 76, 329, 1995.

Wolfe, C.J. and S.C. Solomon, Shear-wave splitting and implications for mantle flow beneath the MELT region of the East Pacific rise, Science, 280, 1230-1232, 1998.

Zhang, S. and S.-I. Karato, Lattice preferred orientation of olivine aggregates deformed in simple shear, Nature, 375, 774-777, 1995. 
Table 1. Peak coherence across the Pacific between Rayleigh wave group speed maps at the indicated periods.

\begin{tabular}{ccccc} 
& $25 \mathrm{sec}$ & $50 \mathrm{sec}$ & $100 \mathrm{sec}$ & $150 \mathrm{sec}$ \\
\hline $25 \mathrm{sec}$ & 1.00 & 0.62 & 0.56 & 0.53 \\
$50 \mathrm{sec}$ & 0.62 & 1.00 & 0.70 & 0.59 \\
$100 \mathrm{sec}$ & 0.56 & 0.70 & 1.00 & 0.82 \\
$150 \mathrm{sec}$ & 0.53 & 0.59 & 0.82 & 1.00
\end{tabular}



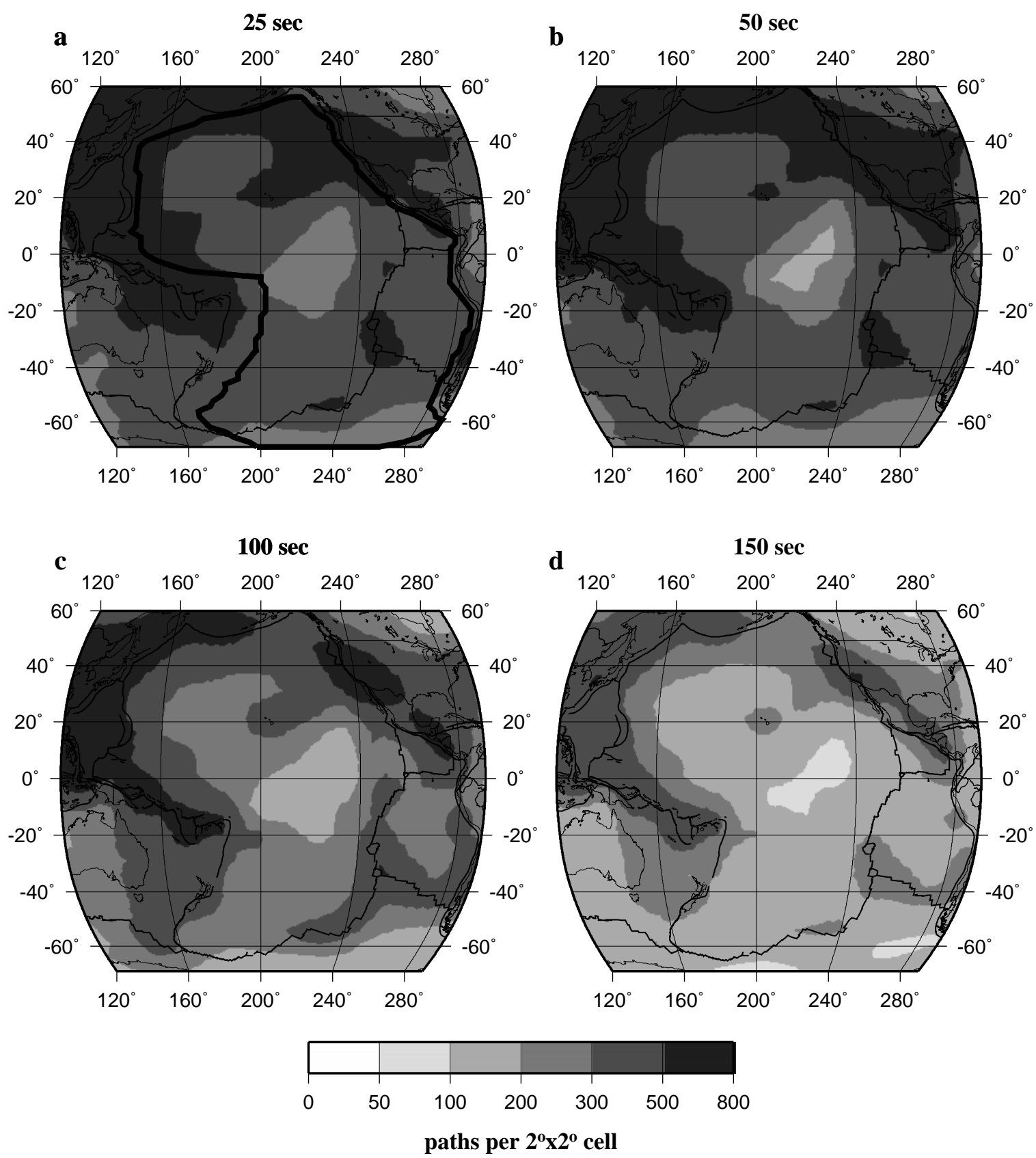

Figure 1. Path density for Rayleigh wave group velocities at the periods indicated. Path density is defined as the number of measurement paths crossing each $2^{\circ} \times 2^{\circ}$ cell $(\sim 50,000$ $\mathrm{km}^{2}$ ). In (a), the thick contour defines the area that we refer to as the "Pacific". 


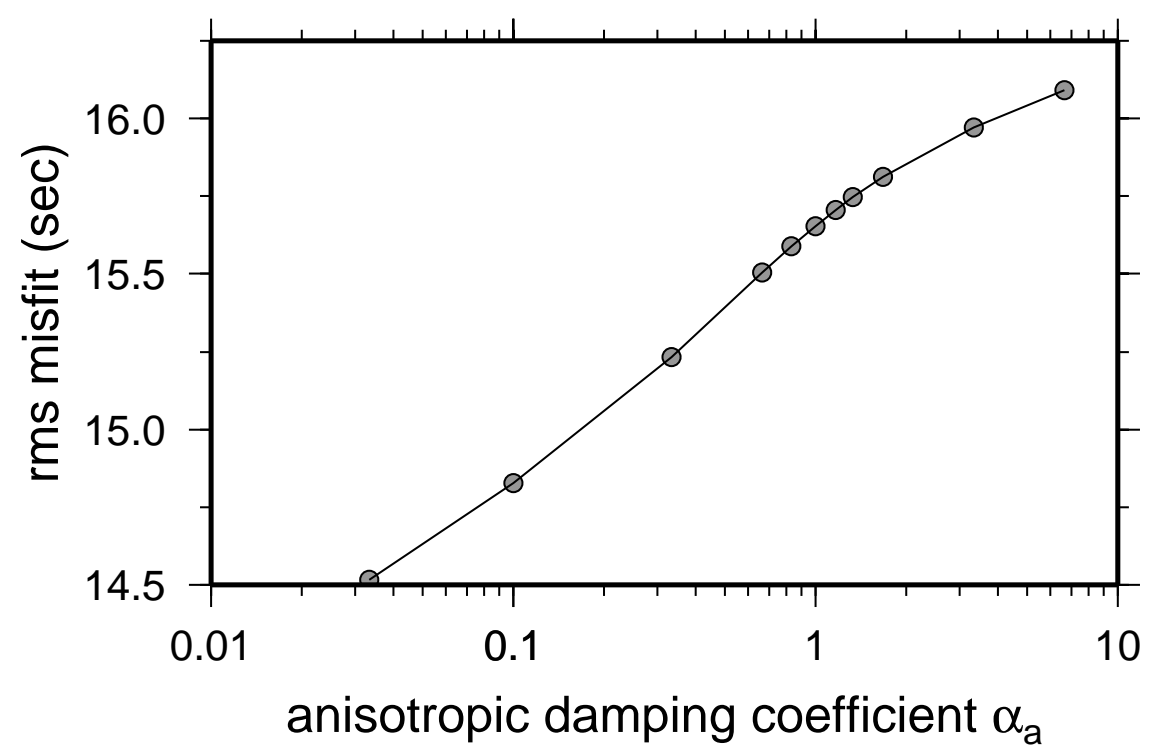

Figure 2. Misfit to $50 \mathrm{sec}$ Rayleigh wave group speed measurements from paths across the Pacific plotted versus a broad-range of values of the anisotropy damping parameter $\alpha_{a}$. Misfit is defined as the rms-difference between the measured surface wave travel times and the travel times predicted by the estimated model, in seconds. 


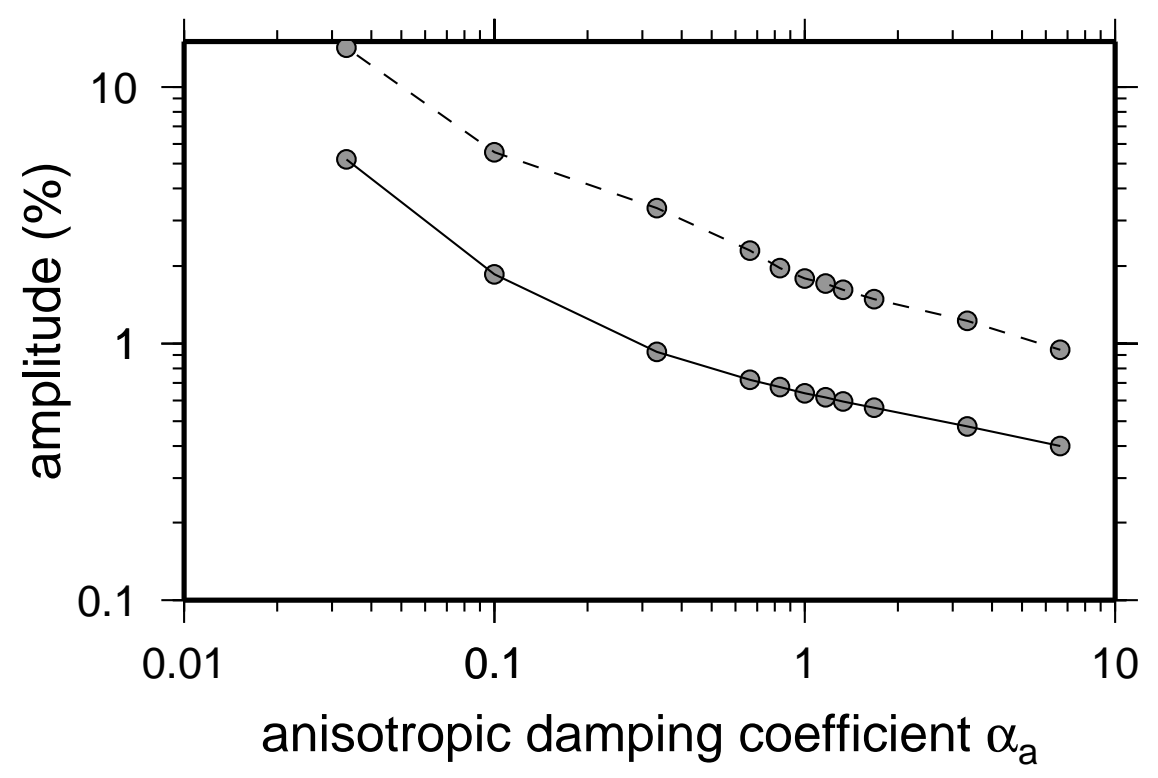

Figure 3. Amplitude of azimuthal anisotropy for the 50 sec Rayleigh wave group speed (eq. (4)) plotted versus a broad-range of values for the anisotropy damping parameter $\alpha_{a}$. The solid line is the average across the Pacific and the dashed line is the maximum amplitude of anisotropy in the Pacific. There is, on average, about a factor of 3 between the average and maximum values. 


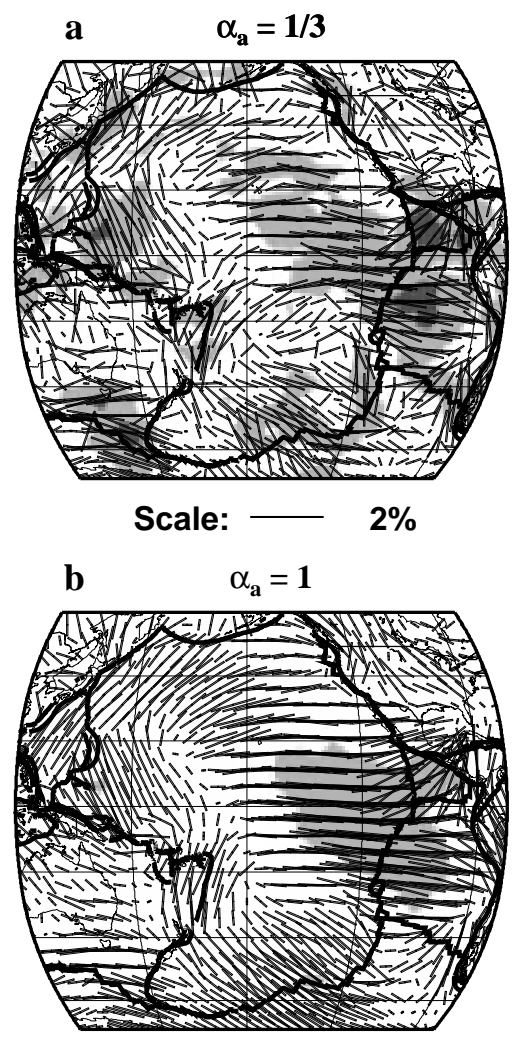

Scale: $-1 \%$

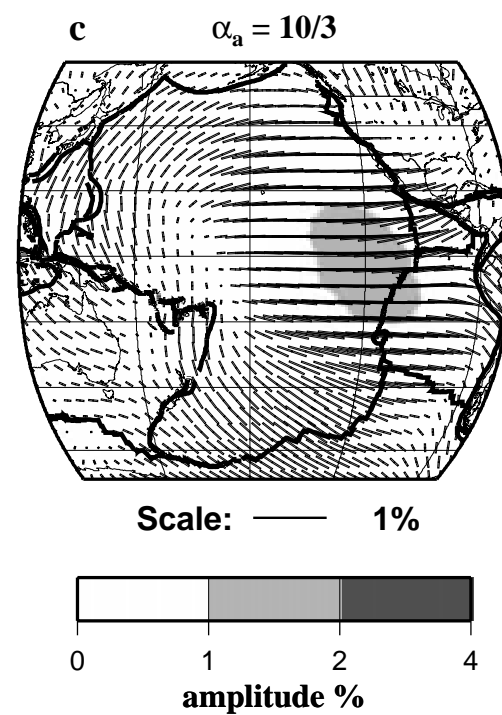

Figure 4. The $2 \psi$ component of azimuthal anisotropy for 50 sec Rayleigh wave group speed estimated using three different values of the anisotropy damping parameter $\alpha_{a}: 1 / 3,1,10 / 3$. Line segments show the fast axis direction with the length proportional to the percent deviation from the reference velocity. Amplitudes, defined by equation (4), are also shown with greyshading. 

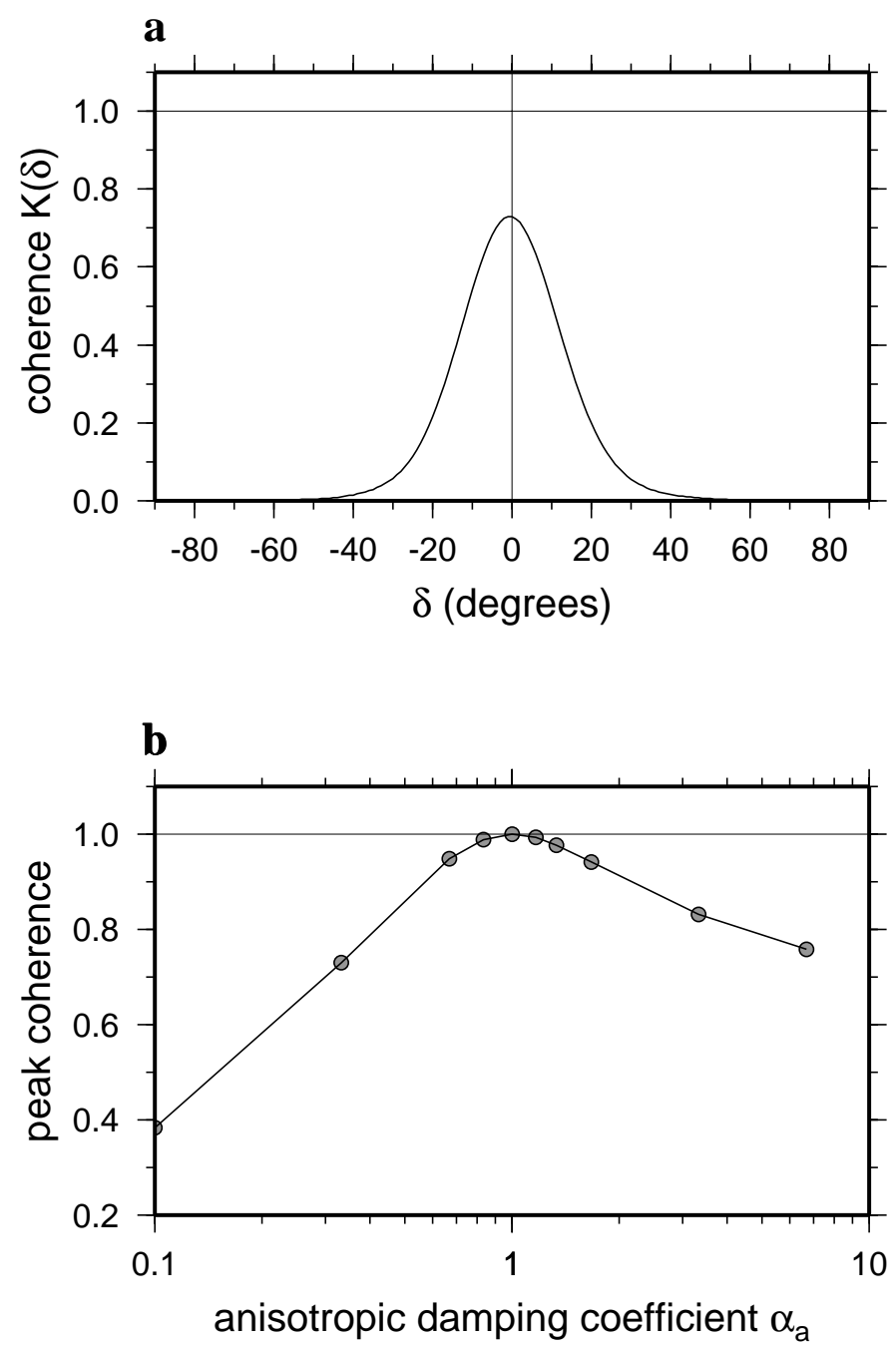

Figure 5. Spatial coherence (eq. (5)) across the Pacific of Rayleigh wave group speed azimuthal anisotropy at $50 \mathrm{sec}$ period for different values of the anisotropic damping parameter $\alpha_{a}$. (a) Coherence between anisotropy estimated with $\alpha_{a}=1$ and $\alpha_{a}=1 / 3$. (b) Peak coherence between anisotropy estimated with $\alpha_{a}=1$ and a broad range of different values of $\alpha_{a}$. Peak coherence is the maximum value of coherence, approximately equal to the y-intercept on plots such as those in (a). 

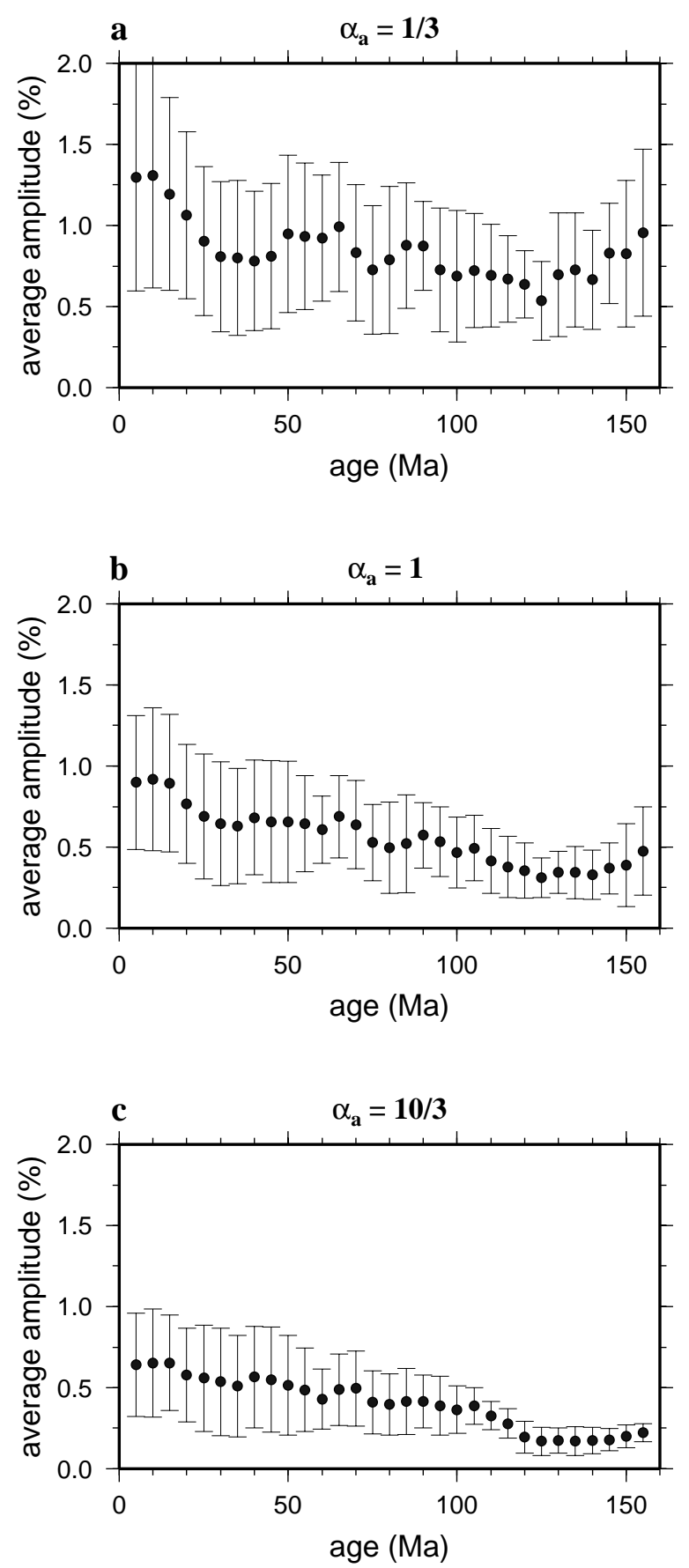

Figure 6. Amplitude of azimuthal anisotropy (eq. (4)) for the 50 sec Rayleigh wave group speed averaged across the Pacific and plotted versus lithospheric age (Mueller et al., 1997) for three different values of the anisotropy damping parameter: $\alpha_{a}=1 / 3,1,10 / 3$. The "error bars" represent the standard deviation of the amplitudes within each age bin and the black dots are the means. 



Figure 7. The $2 \psi$ component of azimuthal anisotropy for Rayleigh wave group velocities across the Pacific. $\left(\sigma_{i}=300 \mathrm{~km}, \alpha_{i}=4 / 3, \sigma_{a}=10^{3} \mathrm{~km}, \alpha_{a}=1\right.$.) Line segments are the fast axis directions whose length is proportional to the amplitude of anisotropy (eq. (4))) presented as a percent deviation from a reference velocity. Amplitudes, defined by equation (4), are shown with grey-shading as in Figure 4. 

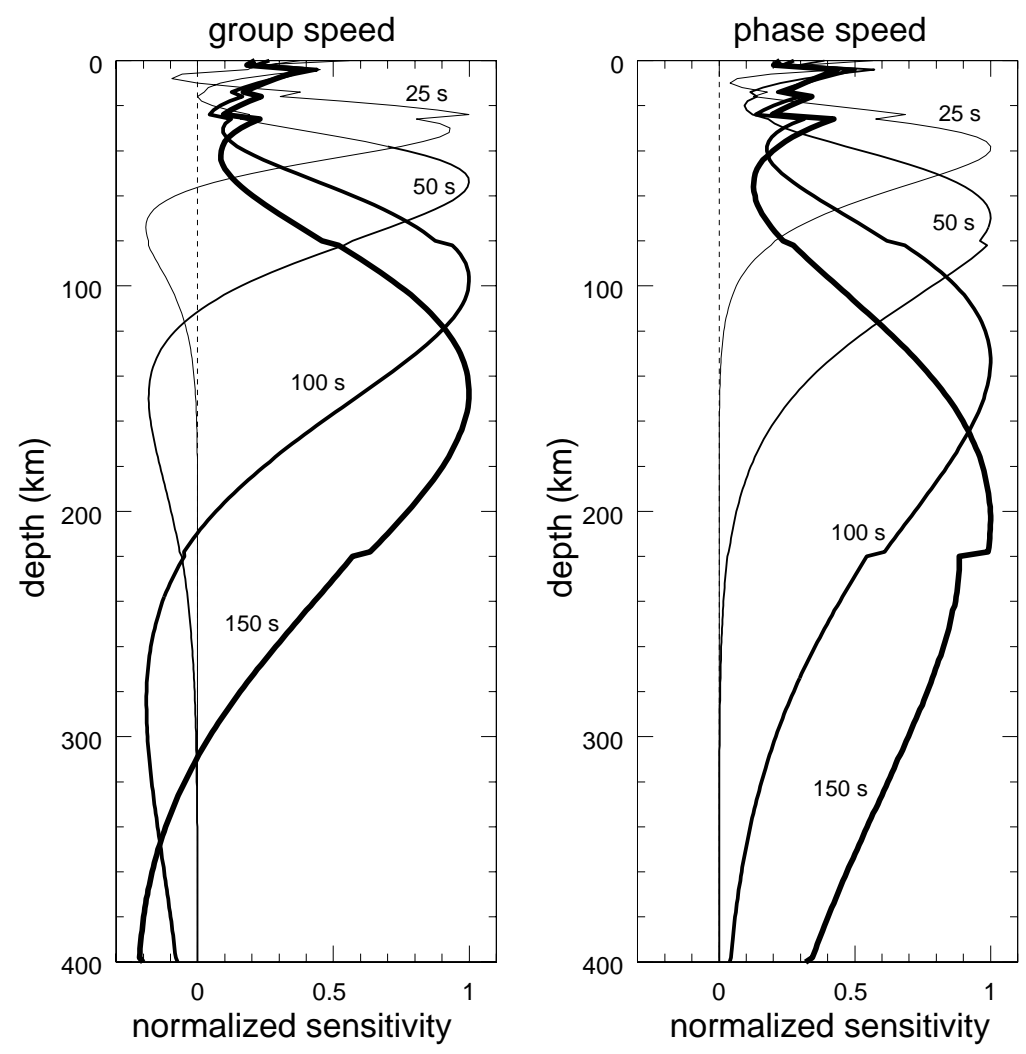

Figure 8. Sensitivity kernels for fundamental mode Rayleigh waves. (LEFT) Group velocity sensitivity kernels at $25 \mathrm{sec}, 50 \mathrm{sec}, 100 \mathrm{sec}$, and $150 \mathrm{sec}$ periods. (RIGHT) Phase velocity sensitivity kernels at at the same periods. The kernels are amplitude normalized, have units of inverse length, and are defined as $\partial c / \partial v_{s}$ at a constant frequency, where $c$ is group or phase speed, and $v_{s}$ is shear wave speed. 


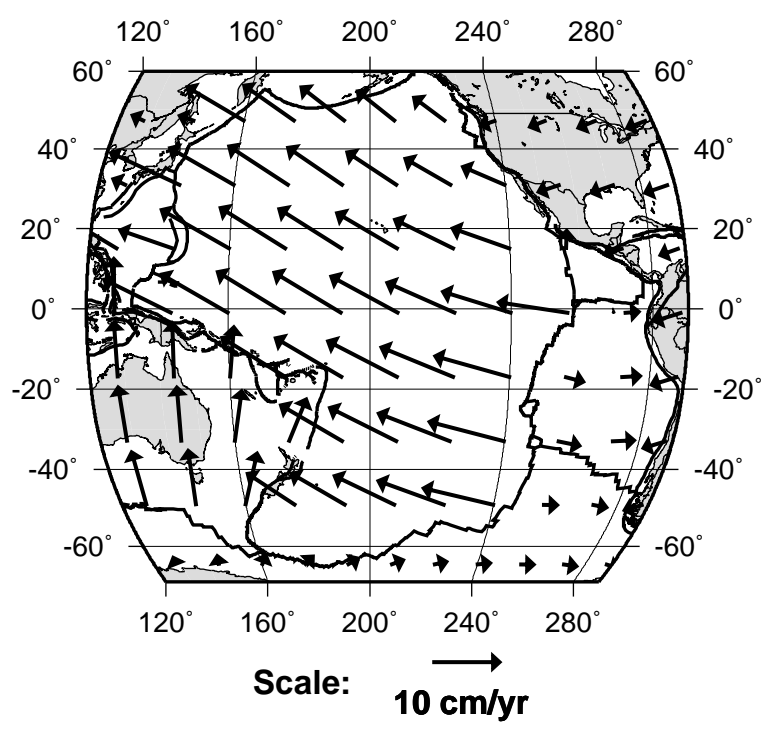

Figure 9. (a) Present-day plate motions defined in the hot-spot frame using the HS3-NUVEL1A model [Gripp \& Gordon, 2002]. 

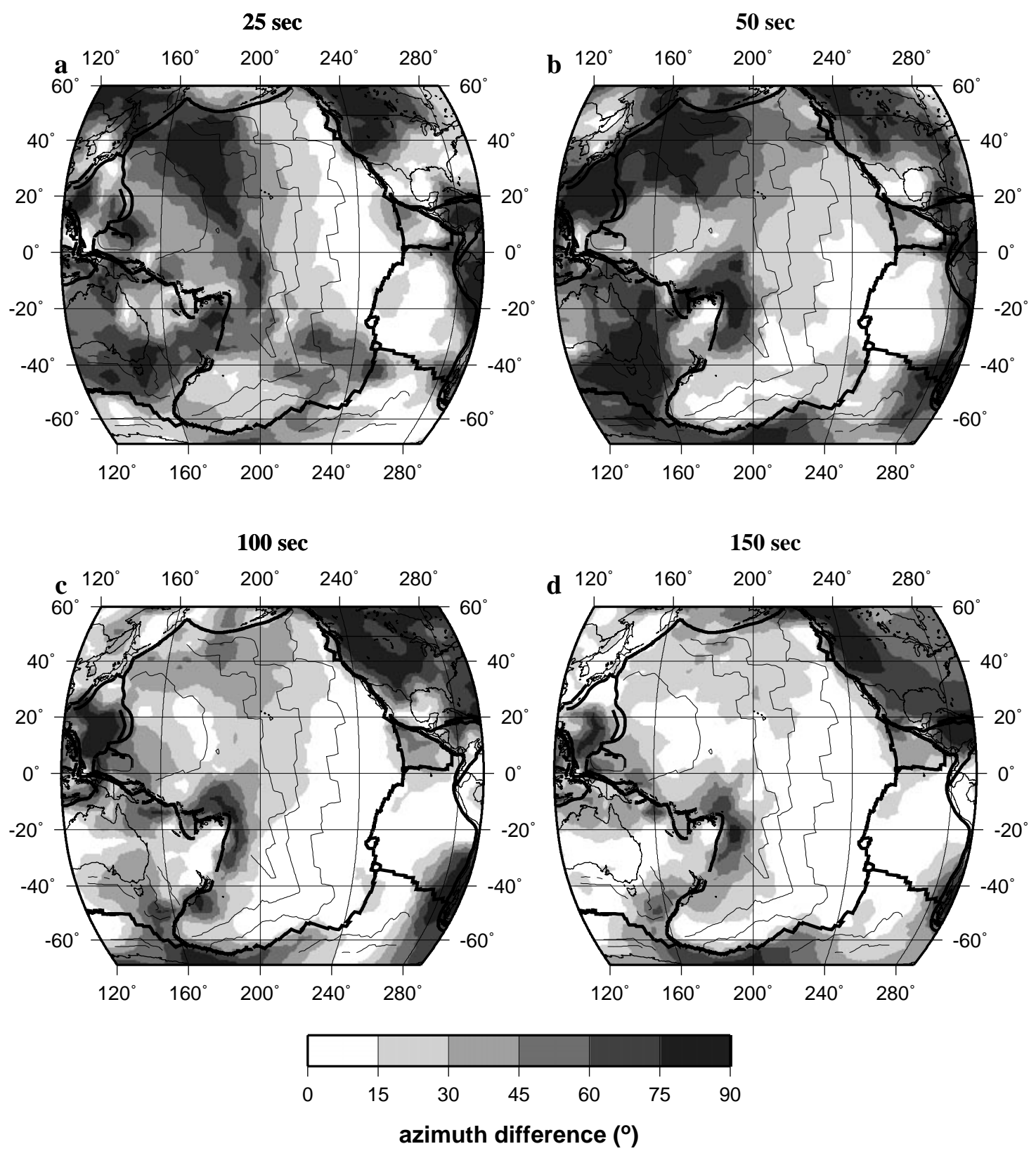

Figure 10. Difference in azimuth between the $2 \psi$ component of azimuthal anisotropy (Fig. 7) and present-day plate motion (Fig. 9). In the young Pacific, the fast axes at all periods lie approximately parallel to plate motion except in the southern Pacific at $25 \mathrm{sec}$ and in the Antarctic Plate. Even in the old Pacific the fast axes at $150 \mathrm{sec}$ lie approximately parallel to plate motion. At shorter periods in the old Pacific, however, fast axes increasingly diverge from the direction of plate motion. Isochrons of lithospheric age (Mueller et al., 1997) in $35 \mathrm{Ma}$ increments are plotted for reference. 

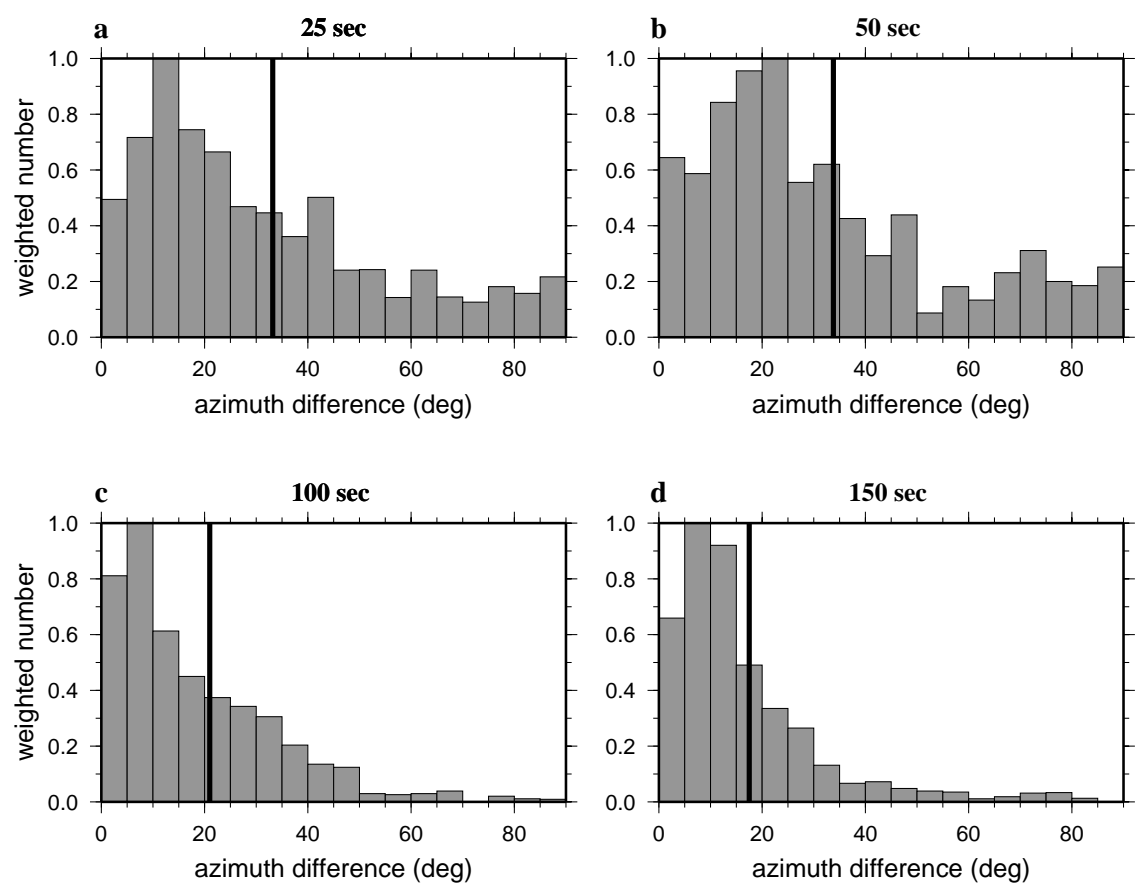

Figure 11. Normalized histograms of the difference in azimuth between the $2 \psi$ fast axis directions and present-day plate motions for Rayleigh wave group speed at the indicated periods. The vertical line in each panel shows the weighted mean of the difference across the Pacific. 

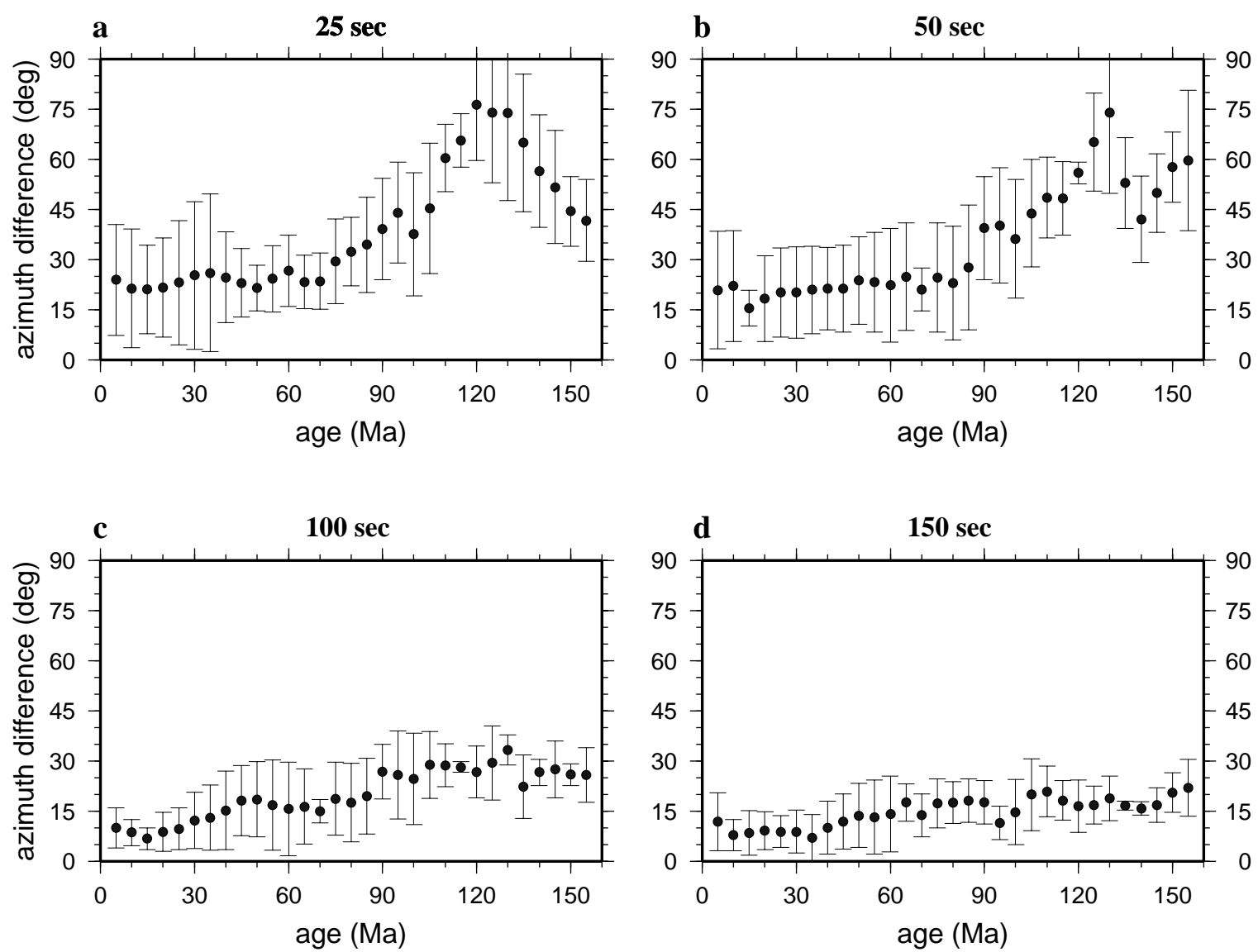

Figure 12. Difference in azimuth between anisotropic fast axis directions and the directions of plate motion plotted versus lithospheric age of the Pacific. The "error bars" are defined as in Figure 6. At young ages the fast axes at all periods align approximately parallel to plate motion. At old ages, the fast axes at long periods $(\mathrm{c}, \mathrm{d})$ tend to align parallel to plate motion, but for short periods $(\mathrm{a}, \mathrm{b})$ the fast axes diverge from plate motion directions. Each datum is weighted as in Figure 11. 


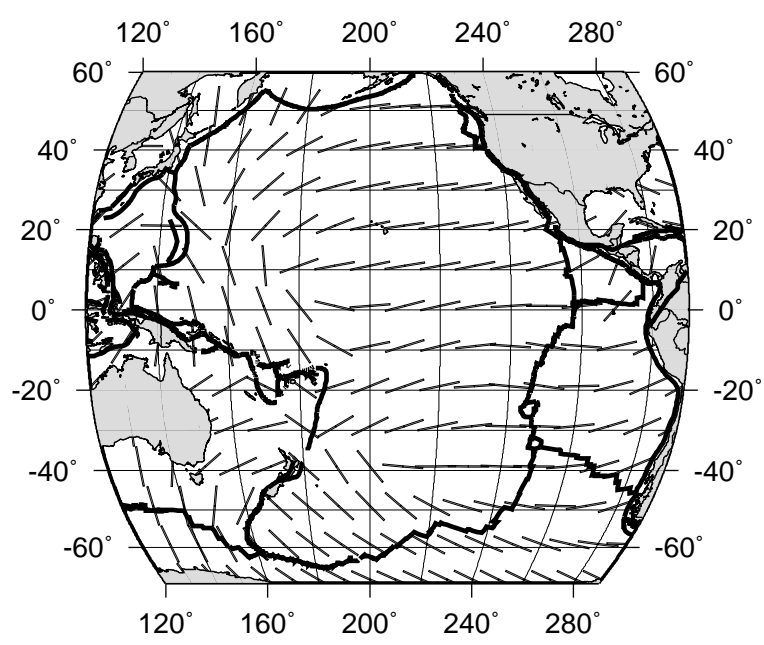

Figure 13. Estimated paleo-spreading directions computed from the gradient of lithospheric age (Mueller et al., 1997). 

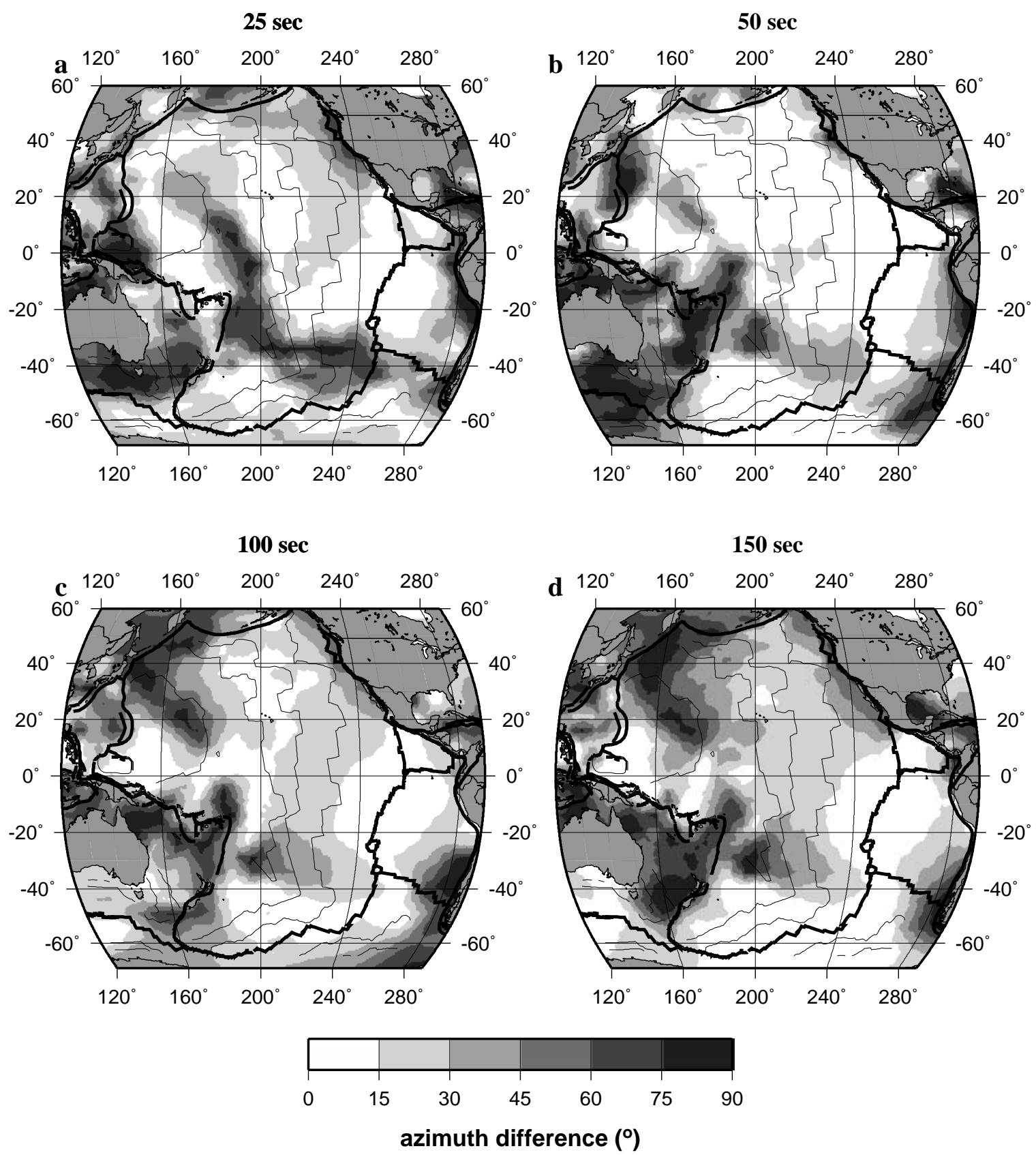

Figure 14. Same as Fig. 10, but this is the difference in azimuth between the $2 \psi$ component of azimuthal anisotropy and the paleo-spreading direction. Agreement is best at 50 sec period and degrades as period increases. 

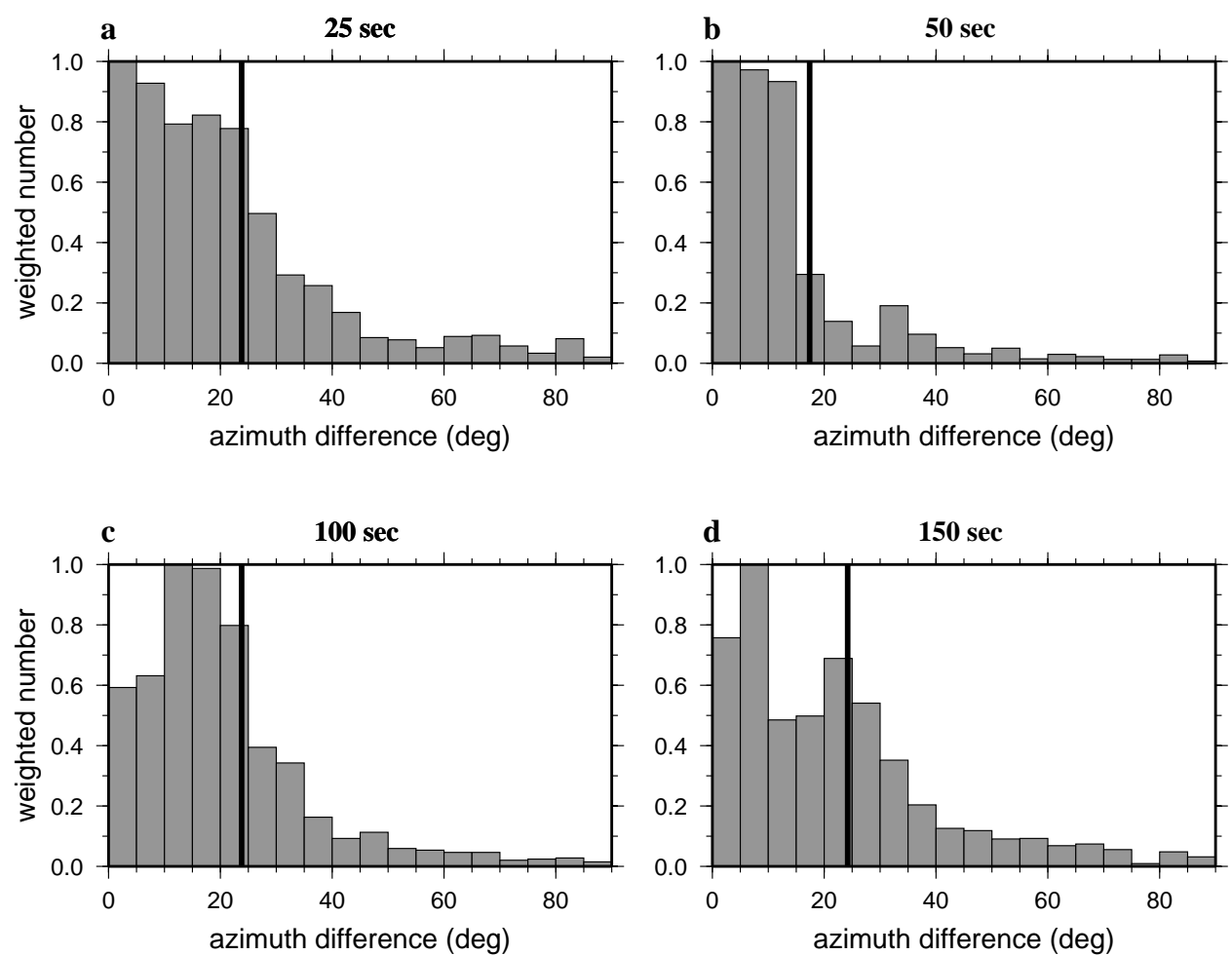

Figure 15. Same as Fig. 11, but these are histograms of differences in azimuth between the $2 \psi$ component of azimuthal anisotropy and the paleo-spreading direction. 

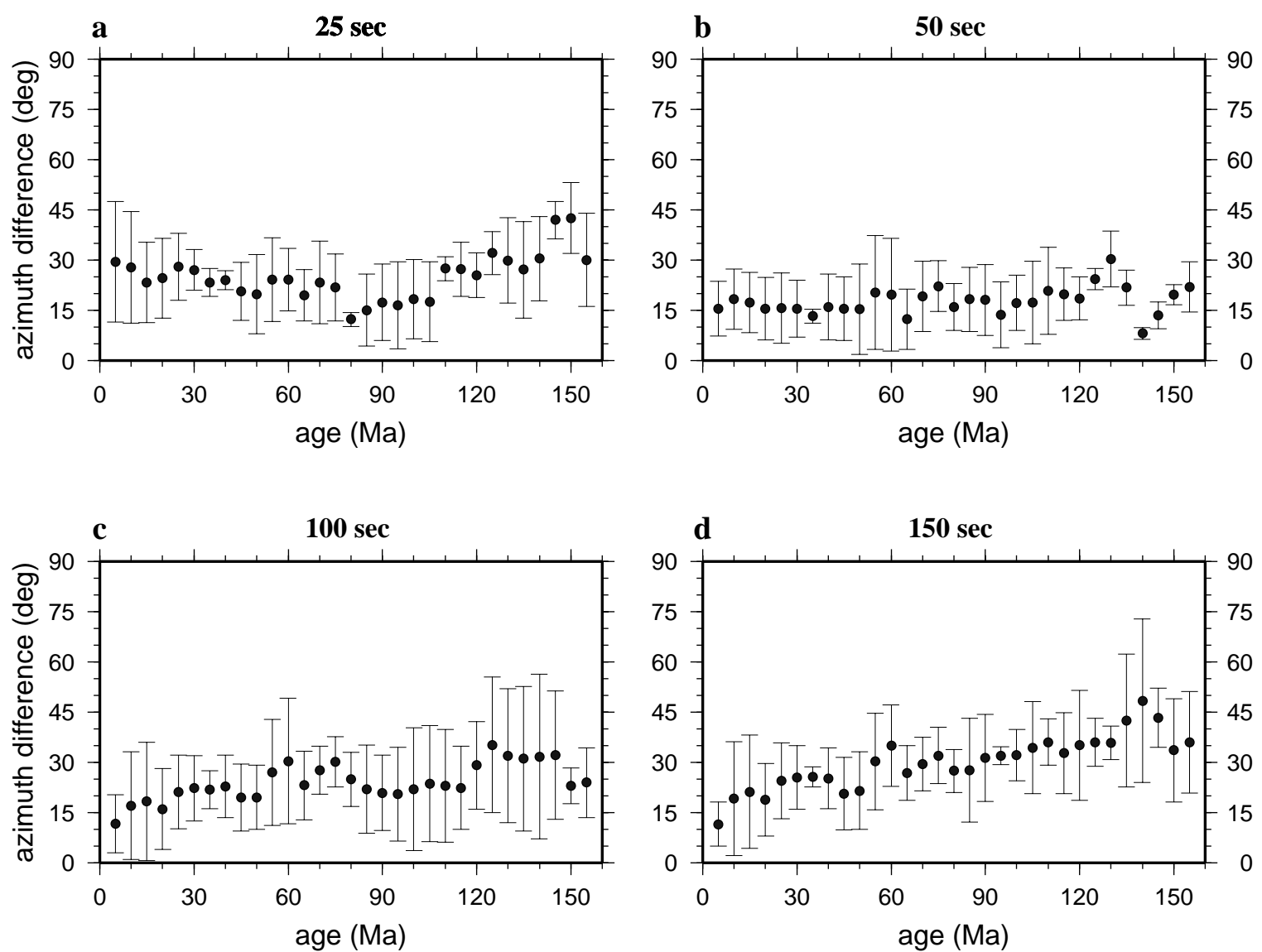

Figure 16. Same as Figure 12, but this is the difference between anisotropic fast axis directions and paleo-spreading directions. At young ages the fast axes approximately align parallel to the paleo-spreading directions. At old ages, the fast axes at short periods $(\mathrm{a}, \mathrm{b})$ align nearer to the paleo-spreading directions than the fast axes for long periods $(\mathrm{c}, \mathrm{d})$. Each data point is weighted in the same manner as in Figure 11. 


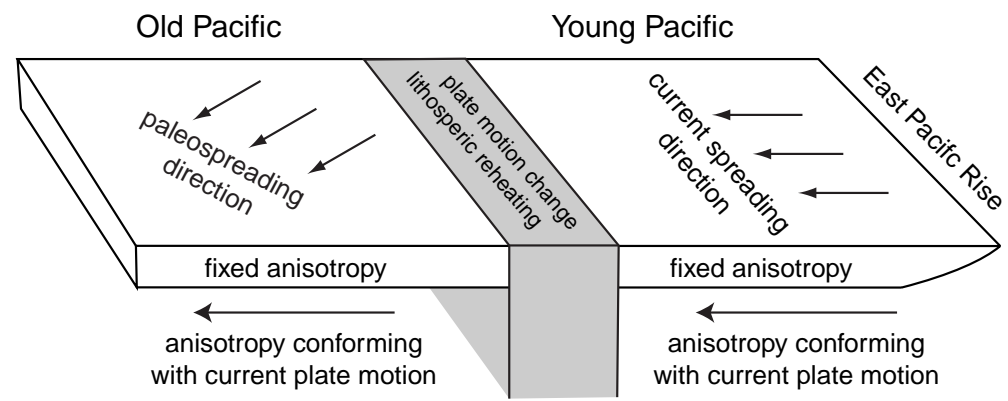

Figure 17. Two layer stratification of the average structure of azimuthal anisotropy in the Pacific upper mantle. In the asthenosphere and deep lithosphere, azimuthal anisotropy is able to conform to contemporary conditions and aligns approximately parallel to present-day plate motions. In the shallow lithosphere, the fast axes are fixed and align approximately parallel to the direction of spreading at the time of lithospheric formation. 

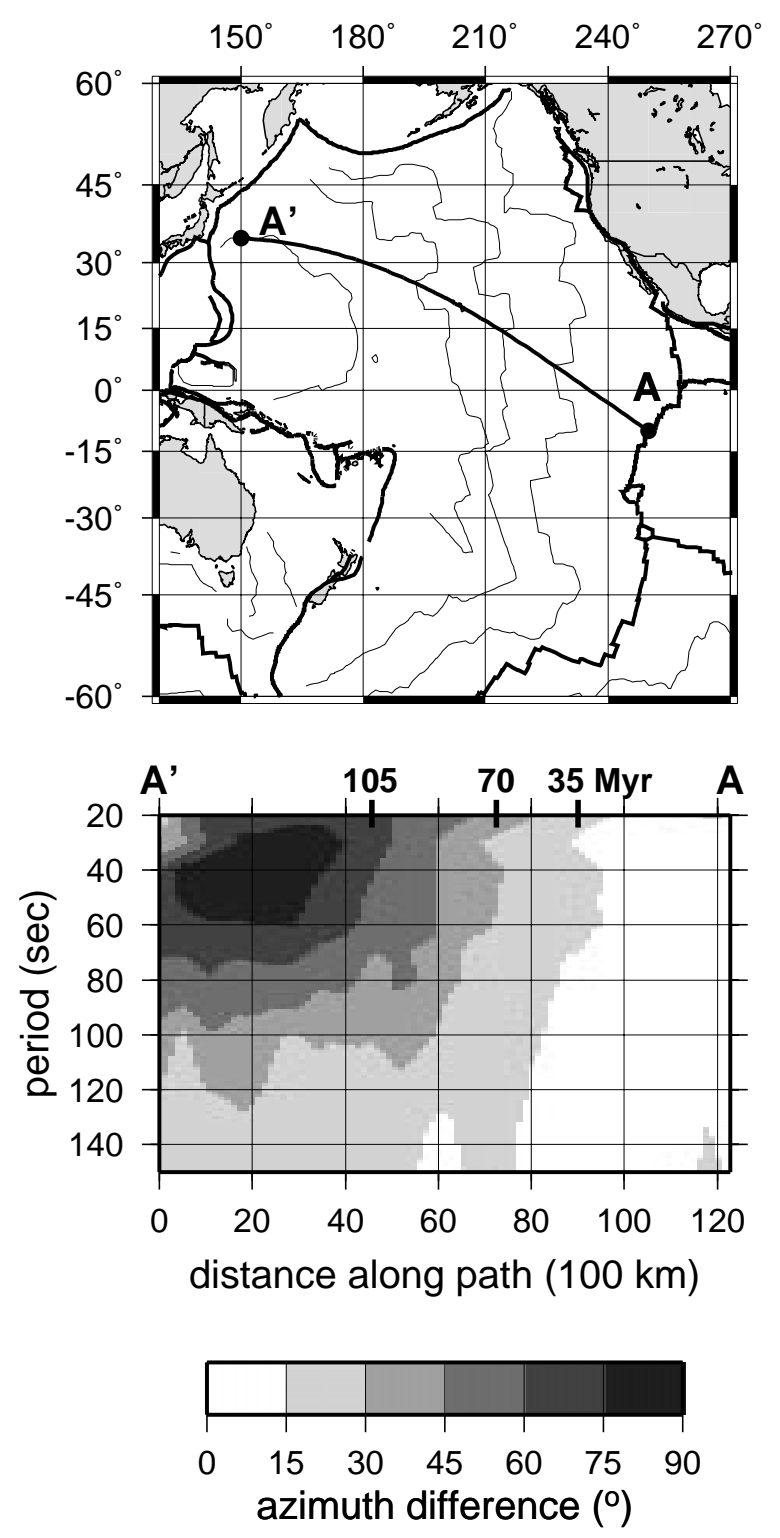

Figure 18. The period dependence (bottom) of the difference in azimuth between anisotropic fast axis directions and present-day plate motions along a great circle path across the Pacific (top). Because the period of the Rayleigh wave is an approximate proxy for depth of sensitivity (e.g., Fig. 8), this gives an idea of the depth variation of the fast axis directions relative to plate motions. 

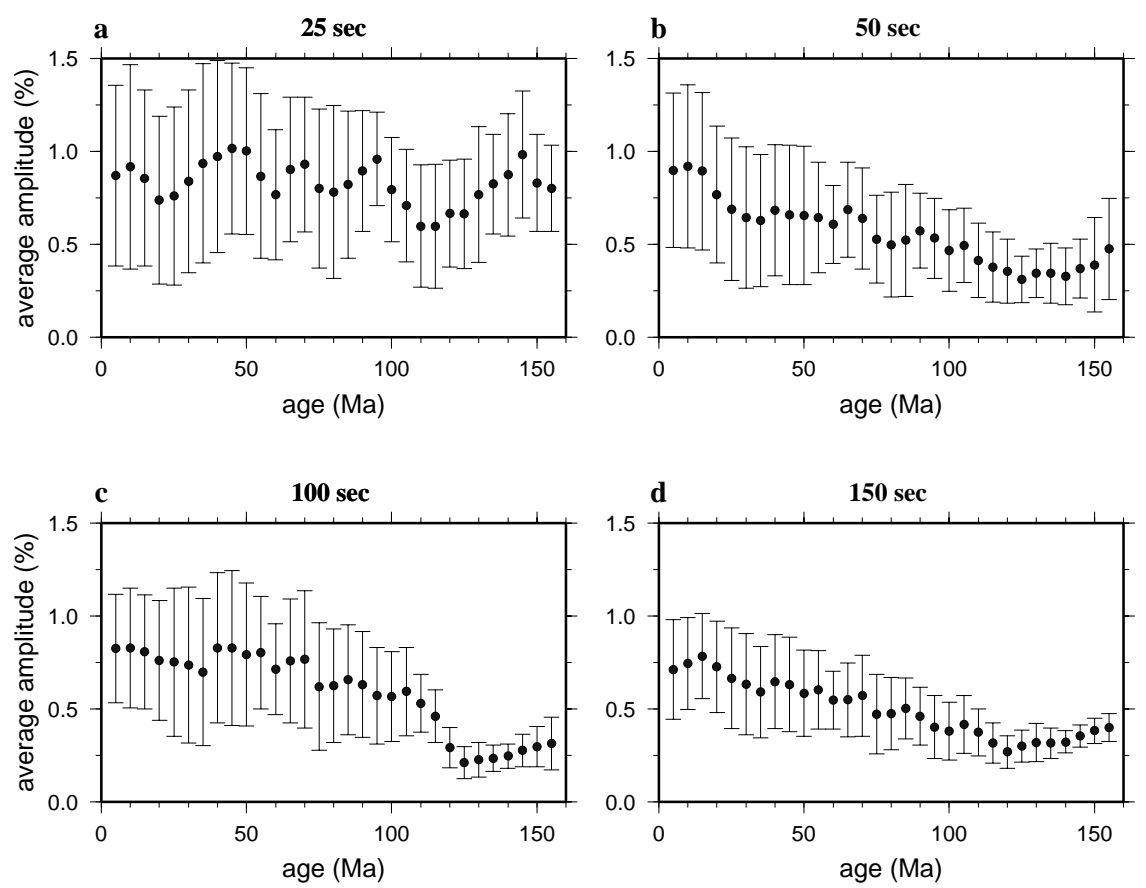

Figure 19. The amplitude of azimuthal anisotropy (eq. (4)) as a function of period and lithospheric age. At intermediate and long periods (50 - $150 \mathrm{sec}$ ), amplitudes decrease systematically with lithospheric age. At short periods (25 sec), amplitudes do not display this trend. 


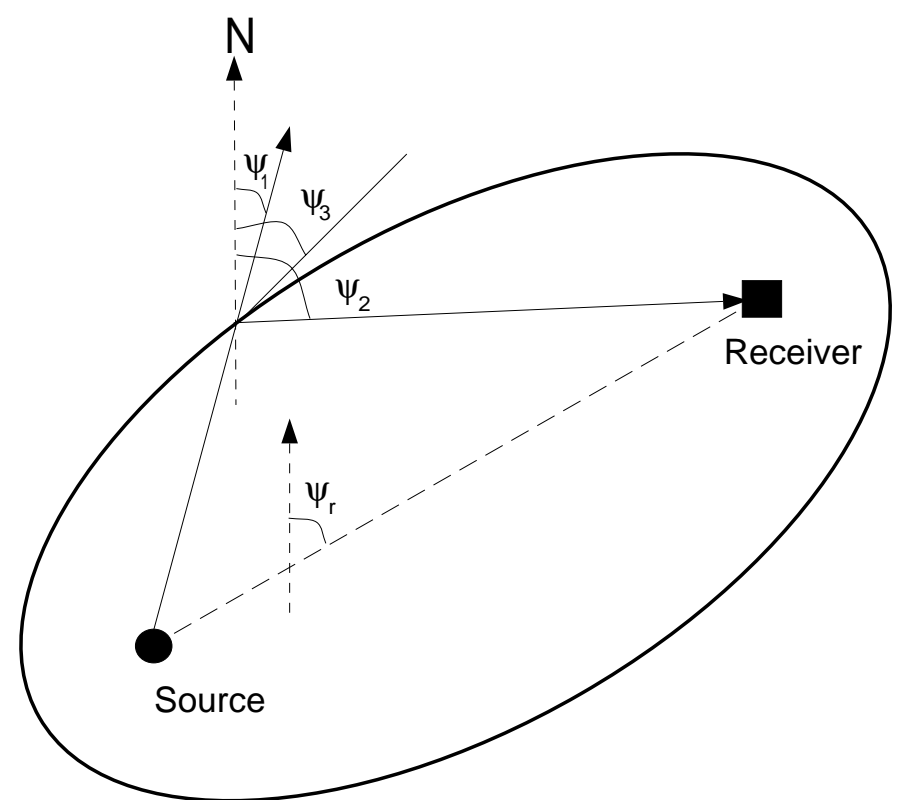

Figure 20. Three definitions of the local azimuth at the scatter: $\psi_{1}$ - azimuth between the source and the scatterer, $\psi_{2}$ - azimuth between the scatterer and the receiver, and $\psi_{3}$ - the average of $\psi_{1}$ and $\psi_{2}$. 

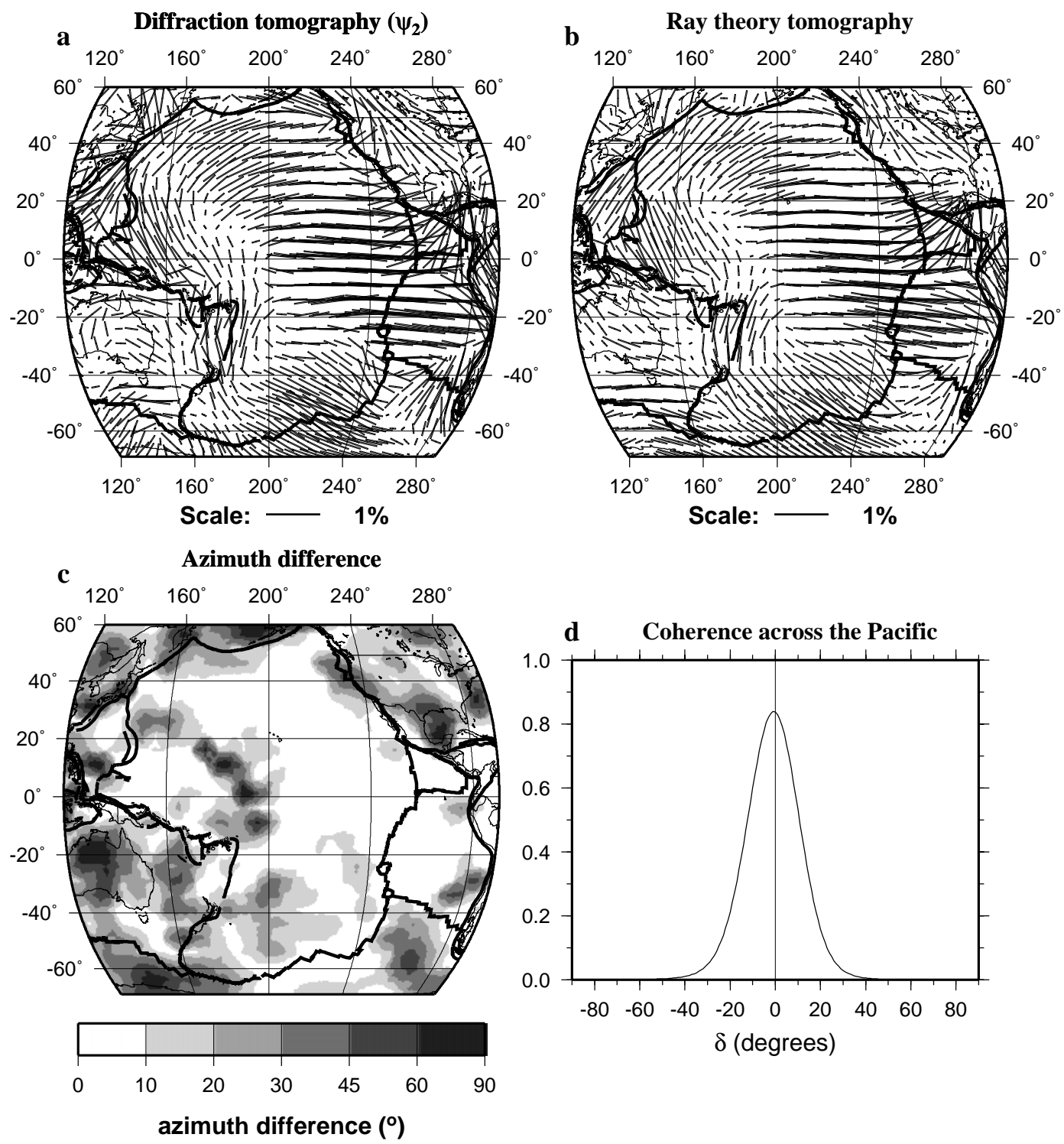

Figure 21. The effect of diffraction through the anisotropic model on estimates of azimuthal anisotropy for the $50 \mathrm{sec}$ Rayleigh wave group speed. (a) Anisotropy results based on diffraction tomography for both the isotropic and anisotropic parts of the model, where the local azimuth at the scatterer is defined as the angle between the scatterer and the receiver $\left(\psi_{2}\right.$ in Fig. 20$)$.

(b) Anisotropy results using ray theory for the anisotropic part of the model and diffraction theory for the isotropic part. (c) Difference in azimuth between the fast axis directions in (a) and (b). The largest differences occur near plate boundaries, on continents, and where the amplitude of anisotropy is small (e.g., in parts of the Central Pacific). (d) Coherence between the results in (a) and (b) across the Pacific. 